\title{
1 MicNet Toolbox: visualizing and deconstructing a microbial
}

\section{2 network}

3

4 Python toolbox for microbial community analysis

5

6 Natalia Favila ${ }^{1 \pi}$, David Madrigal-Trejo ${ }^{2 \pi}$, Daniel Legorreta ${ }^{1}$, Jazmín Sánchez-Pérez ${ }^{2}$, Laura

7 Espinosa-Asuar ${ }^{2}$, Valeria Souza ${ }^{2 *}$

8

$9 \quad{ }^{1}$ Laboratorio de Inteligencia Artificial, Ixulabs. Mexico City, Mexico.

102 Departamento de Ecología Evolutiva, Instituto de Ecología, Universidad Nacional Autónoma de

11 México, AP 70-275, Mexico City 04510, Mexico

12

13

14

${ }^{*}$ Corresponding author

15

E-mail: souza@unam.mx

16

17

18

19

20

I These authors contributed equally to this work.

21

22

23

24

25 


\section{Abstract}

Understanding both global and local patterns in the structure and interplay of microbial communities has been a fundamental question in ecological research. In this paper, we present a python toolbox that combines two emerging techniques that have been proposed as useful when analyzing compositional microbial data. On one hand, we introduce a visualization module that incorporates the use of UMAP, a recent dimensionality reduction technique that focuses on local patterns, and HDBSCAN, a clustering technique based on density. On the other hand, we have included a module that runs an enhanced version of the SparCC code, sustaining larger datasets than before, and we couple this with network theory analyses to describe the resulting co-occurrence networks, including several novel analyses, such as structural balance metrics and a proposal to discover the underlying topology of a co-occurrence network. We validated the proposed toolbox on 1) a simple and well described biological network of kombucha, consisting of 48 ASVs, and 2) using simulated community networks with known topologies to show that we are able to discern between network topologies. Finally, we showcase the use of the MicNet toolbox on a large dataset from Archean Domes, consisting of more than 2,000 ASVs. Our toolbox is freely available as a github repository (https://github.com/Labevo/MicNetToolbox), and it is accompanied by a web dashboard (http://micnetapplb-1212130533.us-east-1.elb.amazonaws.com) that can be used in a simple and straightforward manner with relative abundance data.

\section{Author Summary}

Microbial communities are complex systems that cannot be wholly understood when studied by its individual components. Hence, global pattern analyses seem to be a promising complement to highly focused local approaches. Here, we introduce the MicNet toolbox, an open-source collection of several analytical methods for visualizing abundance data and creating co-occurrence networks for further analysis. We include two modules: one for visualization and one for network analysis 
based on graph theory. Additionally, we introduce an enhanced version of SparCC, a method to estimate correlations for co-occurrence network construction, that is faster and can support larger datasets. We performed method validations using simulated data and a simple biological network. Our toolbox is freely available in a github repository at https://github.com/Labevo/MicNetToolbox, and it is accompanied by a web dashboard that could be easily accessed and manipulated by nonspecialist users. With this implementation, we attempt to provide a simple and straightforward way to explore and analyze microbial relative abundance data.

\section{Introduction}

Microbiomes are not a mere collection of independent individuals, but rather, ensembles of intricate constituents, biotic and abiotic, that create highly complex systems where emergent interactions, structures, and functions are crucial for the survival and performance of the whole. The inference of microbial co-occurrence networks may help in understanding emergent properties of these systems [1]: unravelling microbial interactomes [2], evaluating the effects of stress and perturbations in community stability [3], and providing a wide array of novel applications including diagnostics of environmental quality [4] and pathogen identification in disease management [5].

One promising contribution to unravel microbial ecological associations has been the application of network science as an increasingly used alternative to study complex systems [6], whose methods can handle the scale and diversity of high-throughput biological data [1]. Several approaches have been developed to infer microbial ecological associations, such that patterns can be visualized and analyzed within the schematic of a network. One of the most used family of methods is the inference by co-occurrence and correlations, such as: Pearson [7] or Spearman [8] correlation coefficient, Jaccard distance [9] or Bray-Curtis dissimilarity [10], Local Similarity Analysis [11,12], Maximal Information Coefficient [13], MENA that adapts Random Matrix Theory [14,15], SparCC based on Aitchison's log-ratio analysis [16,17], and CoNet which combines information of several metrics $[18,19]$. Other types of techniques, such as ordinary differential 
equations (ODE) models [20] have also been used as an alternative to capture microbial interactions, amongst which the generalized Lotka Volterra equations (gLT) are one of the most used [21] for two-species systems, and potentially useful for three-species systems or larger [22]. Finally, MetaMIS [23], LIMITS [24], and some variations which integrate forward stepwise regressions and bootstrap aggregation [2], offer a different implementation of the gLV equations.

Although the potential value of microbial co-occurrence networks is known, there are several caveats and limitations. To start with, high-throughput genomic data is often associated with low annotation resolution at the species level, which makes it difficult to differentiate between strains and species. This has led to the usage of ASVs (Amplicon Sequence Variants) or OTUs (Operational Taxonomic Units) to obtain a more reliable account of discrete ecological players, leading to hundreds and sometimes thousands of potential organisms [5,25]. However, there are just a few techniques which enable the use of thousands of OTUs/ASVs in the construction of networks for the most diverse ecosystems, such as soil [19]. Furthermore, microbial abundances are normally presented as relative abundance matrices, which creates compositional data sets that are often sparse [1]. Some existing methods are commonly known to provide an efficient approach for compositional effects, spurious correlations, and sparse data handling; nonetheless, biological interaction inferences from compositional data alone should be taken with caution (see Weiss et al. (2016) [22], Dohlman \& Shen (2019) [2], Hirano \& Takemoto (2019) [26] for a review on performance). Finally, noise or contamination should be expected not be part of the real system captured in the network and the identification of taxa that are not an integral part of a community could be fundamental for more accurate biological interpretations [22].

These issues have led to a high divergence of results when trying to infer direct correlations between OTUs/ASVs [1], therefore algorithms with a more reliable statistical approach are needed. In addition to the intrinsic limitations of inferring interactions from microbial community data, there is a gap in the analysis of networks to obtain the most biological-relevant information: many of the existing methodologies are not easily reachable to the research community, nor do they implement posterior analysis to retrieve information of the co-occurrence network in a clearly and accessible format. Furthermore, at the interpretation level, biologically-meaningful inferences derived co- 
bioRxiv preprint doi: https://doi.org/10.1101/2021.11.11.468289; this version posted November 11, 2021. The copyright holder for this preprint (which was not certified by peer review) is the author/funder, who has granted bioRxiv a license to display the preprint in perpetuity. It is made available under aCC-BY 4.0 International license.

Table 1. Description of several network metrics and properties currently used in biological

113 networks, including some of their prospective interpretations.

\begin{tabular}{|c|c|c|c|}
\hline $\begin{array}{l}\text { Metric/Network } \\
\text { property }\end{array}$ & Definition & $\begin{array}{l}\text { Prospective biological Interpretation } \\
\text { in microbial co-occurrence networks }\end{array}$ & References \\
\hline Total Nodes/Vertices & Total Entities within a network. & $\begin{array}{l}\text { Total number of taxa (species, } \\
\text { OTUs/ASVs) in a network (species } \\
\text { richness); number of connected taxa; } \\
\text { common measure of ecosystem state in } \\
\text { response to perturbations }\end{array}$ & {$[4,28-30]$} \\
\hline $\begin{array}{l}\text { Edges, Links, } \\
\text { Relationship, } \\
\text { connection }\end{array}$ & $\begin{array}{l}\text { Relationship or associations } \\
\text { between nodes. For co-occurrence } \\
\text { networks, relationships exist } \\
\text { between pairs of nodes. }\end{array}$ & $\begin{array}{l}\text { Ecological associations, including } \\
\text { interspecific interactions, niche overlap, } \\
\text { cross-feeding, abiotic co-occurrence } \\
\text { drivers, among others }\end{array}$ & {$[1,4,31,32]$} \\
\hline $\begin{array}{l}\text { Density, } \\
\text { Connectance, } \\
\text { Complexity (network } \\
\text { scale), Interactions } \\
\text { diversity, Probability } \\
\text { of connection }\end{array}$ & $\begin{array}{c}\text { Fraction of edges that are actually } \\
\text { present in the network with respect } \\
\text { to all possible edges. }\end{array}$ & $\begin{array}{c}\text { Reflection of the incidence of } \\
\text { ecosystemic processes; Possible } \\
\text { measure of ecological resilience; } \\
\text { organization level of the community; } \\
\text { measure of complexity in the microbial } \\
\text { network }\end{array}$ & {$[4,28,33-36]$} \\
\hline Connectivity & $\begin{array}{c}\text { Total number of relationships in a } \\
\text { network }\end{array}$ & $\begin{array}{l}\text { Total number of ecological associations } \\
\text { within a biological network }\end{array}$ & {$[28,37]$} \\
\hline $\begin{array}{l}\text { Connected } \\
\text { component }\end{array}$ & $\begin{array}{l}\text { Sets of nodes, where every pair of } \\
\text { nodes have a path between them. }\end{array}$ & $\begin{array}{l}\text { Microbial network where every } \\
\text { OTU/ASV have an indirect ecological } \\
\text { association with every other OUT/ASV }\end{array}$ & {$[27,38-40]$} \\
\hline $\begin{array}{c}\text { Average degree, } \\
\text { Complexity (taxon } \\
\text { scale), } \\
\text { Connectedness } \\
\text { (normalized degree) }\end{array}$ & $\begin{array}{c}\text { Average number of edges } \\
\text { connected to a node; average } \\
\text { number of neighbors for a given } \\
\text { node. }\end{array}$ & $\begin{array}{c}\text { Measure of complexity in the microbial } \\
\text { network }\end{array}$ & {$[4,27,35,36,41]$} \\
\hline
\end{tabular}


bioRxiv preprint doi: https://doi.org/10.1101/2021.11.11.468289; this version posted November $11,2021$. The copyright holder for this preprint (which was not certified by peer review) is the author/funder, who has granted bioRxiv a license to display the preprint in perpetuity. It is made available under aCC-BY 4.0 International license.

\begin{tabular}{|c|c|c|c|}
\hline Degree centrality & $\begin{array}{l}\text { Centrality of a node based on } \\
\text { degree. i.e., nodes with higher } \\
\text { degree are more central to the } \\
\text { network. It is a measurement of } \\
\text { popularity. }\end{array}$ & $\begin{array}{l}\text { Keystone taxa; taxa that interacts the } \\
\text { most within the community }\end{array}$ & {$[27,36,42,43]$} \\
\hline Closeness centrality & $\begin{array}{c}\text { Centrality of a node based on its } \\
\text { proximity to all other nodes in the } \\
\text { network. It is a measure of } \\
\text { broadcaster nodes, that is, nodes } \\
\text { that can influence the network } \\
\text { fastest }\end{array}$ & $\begin{array}{l}\text { Keystone taxa; taxa that, if perturbed, } \\
\text { influence the network the fastest. }\end{array}$ & {$[27,43,44]$} \\
\hline $\begin{array}{l}\text { Betweenness } \\
\text { centrality }\end{array}$ & $\begin{array}{l}\text { Centrality of a node based on how } \\
\text { often a node is situated on paths } \\
\text { between other nodes. It is a } \\
\text { measurement of bridge nodes }\end{array}$ & $\begin{array}{l}\text { Keystone taxa; taxa more important in } \\
\text { communication in the network. }\end{array}$ & {$[4,27,41,44]$} \\
\hline PageRank & $\begin{array}{l}\text { Centrality measure that computes a } \\
\text { ranking of the nodes based on the } \\
\text { structure of the incoming links. It } \\
\text { identifies hub nodes. }\end{array}$ & Keystone taxa & [45-47] \\
\hline $\begin{array}{l}\text { Negative:Positive } \\
\text { relationship ratio, } \\
\text { Behavior }\end{array}$ & $\begin{array}{l}\text { Ratio of positive and negative } \\
\text { relationships. If }>1 \text { there are more } \\
\text { negative interactions, if }<1 \text { there } \\
\text { are more positive interactions } \\
\text { present in the network. }\end{array}$ & $\begin{array}{l}\text { Potential measure of cooperation level } \\
\text { within the community; measure of } \\
\text { community stability (ecological } \\
\text { resilience and resistance) }\end{array}$ & $\begin{array}{c}{[3,4,36,41,48-} \\
51]\end{array}$ \\
\hline $\begin{array}{l}\text { Average shortest path } \\
\text { length }(A L), \text { Average } \\
\text { geodesic path }\end{array}$ & $\begin{array}{l}\text { Average number of steps in the } \\
\text { shortest paths from one node to } \\
\text { another. It is calculated for all pairs } \\
\text { and then averaged. }\end{array}$ & $\begin{array}{l}\text { Microbial networks usually present small } \\
\text { AL: measure of network's response } \\
\text { speed to perturbations (ecological } \\
\text { resilience); community cohesion; } \\
\text { measure of information and substance } \\
\text { flow }\end{array}$ & $\begin{array}{c}{[4,35,37,42,52-} \\
54]\end{array}$ \\
\hline $\begin{array}{l}\text { Diameter, Longest } \\
\text { geodesic path }\end{array}$ & $\begin{array}{l}\text { Length of the longest finite } \\
\text { geodesic path anywhere in the } \\
\text { network. }\end{array}$ & $\begin{array}{l}\text { Measure of information and substance } \\
\text { flow }\end{array}$ & {$[27,36,42,54]$} \\
\hline $\begin{array}{l}\text { Small world index } \\
\text { (SW) }\end{array}$ & $\begin{array}{l}\text { Index based on a tradeoff between } \\
\text { high clustering coefficient and short } \\
\text { path length, the defining } \\
\text { characteristics of small-world } \\
\text { networks. Networks with SW }>1 \\
\text { are said to have more "small- } \\
\text { worldness". }\end{array}$ & $\begin{array}{l}\text { Microbial network topological property. } \\
\text { Small-world microbial networks } \\
\text { suggests that any two members in the } \\
\text { community could interact with each } \\
\text { other through a few intermediaries. }\end{array}$ & {$[6,55]$} \\
\hline $\begin{array}{l}\text { Clustering coefficient, } \\
\text { Transitivity }\end{array}$ & $\begin{array}{l}\text { Average probability that two nodes } \\
\text { neighbors of a third node are also } \\
\text { connected between each other. }\end{array}$ & $\begin{array}{l}\text { Presence of tripartite relationships (e.g., } \\
\text { higher-order biological interactions) } \\
\text { within the community; possible measure } \\
\text { for redundance. }\end{array}$ & $\begin{array}{c}{[4,27,36,37,42} \\
53]\end{array}$ \\
\hline
\end{tabular}




\begin{tabular}{|c|c|c|c|}
\hline $\begin{array}{c}\text { Modularity, } \\
\text { Assortativity (when } \\
\text { normalized) }\end{array}$ & $\begin{array}{c}\text { Quantification of } \\
\text { compartmentalization into } \\
\text { subgroups. Loosely speaking, high } \\
\text { modularity means that there are } \\
\text { more edges within groups and } \\
\text { fewer between groups. }\end{array}$ & $\begin{array}{c}\text { Modules/Clusters have been interpreted } \\
\text { as niches; shared ecological functions } \\
\text { among taxa; spatial } \\
\text { compartmentalization; silar habitat } \\
\text { preferences; measure of community } \\
\text { stability (ecological resilience and } \\
\text { resistance) }\end{array}$ & $\begin{array}{c}{[1,3,18,27,36,3} \\
7,42,56-60]\end{array}$ \\
\hline $\begin{array}{c}\text { Triad motifs and } \\
\text { Balanced triads } \\
\text { fraction }\end{array}$ & $\begin{array}{c}\text { Motifs are overrepresented } \\
\text { subnetworks (patterns). Triad } \\
\text { motifs are classified by balanced or } \\
\text { imbalanced based on the } \\
\text { relationship types (positive or } \\
\text { negative). }\end{array}$ & $\begin{array}{c}\text { Motifs can be relevant in information } \\
\text { flow (e.g., quorum sensing); potential } \\
\text { biomarkers for microbiome perturbed } \\
\text { state. }\end{array}$ & {$[1,36,61-63]$} \\
\hline
\end{tabular}

In an attempt to capture the most relevant information from a microbial community in their co-occurrence network and to try to overcome some common issues, we have developed the

117 MicNet toolbox, an open source code to create, analyze and visualize microbial co-occurrence 118 networks. We implemented UMAP [64], a dimension reduction algorithm which has been previously 119 used to identify unique clusters of data in several genomic projects [65,66], given that it is both scalable to massive data and able to cope with high diversity [64]. Moreover, we coupled UMAP with different types of projections and HDBSCAN [67], an unsupervised clustering algorithm, able to identify both local and global relationships, as well as filtering out noise. Finally, we used and enhanced version of SparCC, a compositionally aware algorithm, to infer correlations for network construction [17,22]. Additionally, the toolbox includes several analyses of network theory to inspect the topological properties, robustness, structural balance, communities, and hub nodes that arise in microbial co-occurrence networks. The development of the MicNet toolbox, as an 127 integration of several analyses, attempts to provide an easy-to-use and straightforward implementation towards a comprehensive description of potential local and global patterns for a better understanding of microbial community systems. 


\section{Design and Implementation}

\section{Python Implementation}

133 The code of the MicNet toolbox was built using python 3.9 [68]. The MicNet toolbox uses several

134 standard packages in the Python ecosystem for matrices (pandas v1.3.2 [69,70], numpy v1.20.3 135 [71], and dask v2021.8.0 [72]), to improve performance (numba v0.53.1 [73]), for temporary storage 136 (h5py v3.2.1 [74]) and to create visualizations (bokeh v2.3.3 [75]). UMAP and HDBSCAN were 137 implemented using packages umap-learn v0.5.1 [64] and hdbscan v0.8.27 [67], whereas network 138 analyses were performed using functions from the networkx v2.6.2 package [76]. In the following sections we explain the different components implemented in the MicNet toolbox which are summarized in Fig 1.

Fig 1. Overview of MicNet toolbox. MicNet Toolbox was designed for visualizing, creating and analyzing microbial networks obtained from compositional data. It includes a data visualization module which uses UMAP and HDBSCAN to find local patterns in the data, and a network analysis module which implements an enhanced version of SparCC and several network analyses such as topology comparison and community analysis, amongst others.

\section{Input Data}

149 MicNet toobox input data consists of relative abundance/compositional datasets from high150 throughput sequencing methods, such as metagenomics or metabarcoding. Prior to building an 151 abundance data table, raw assembled sequences should be OTU/ASV clustered and taxonomically annotated accordingly. MicNet toolbox currently supports abundance data as .tsv files (separated by tabs) or .csv files (separated by commas). In the web dashboard, input abundance data table is filtered by default, removing singletons ( $<5$ total counts among all samples) and unique (only appearing in one sample) entries. If the user desires, singleton filtering could be deactivated. For 
SparCC and UMAP/HDBSCAN the first column of the table should contain the OTU/ASV ID and

157 the following columns the abundance data. Taxonomic annotation for the given OUT/ASV can be

158 included in the second column if the user wishes to include taxonomic information in the resulting 159 output. In the case of network analyses, the correlation matrix output by SparCC should be input alongside the UMAP/HDBSCAN output datafile.

\section{UMAP and HDBSCAN Implementation}

A common first step when visualizing high-dimensional data is applying a dimensionality reduction technique. In this toolbox, we implemented UMAP (Uniform Manifold Approximation and Projection), a non-linear dimension reduction technique that favors local data preservation, rather than global data, allowing a better identification of finer scale patterns [64]. We coupled UMAP with HDBSCAN (Hierarchical Density-Based Spatial Clustering of Applications with Noise), a hierarchical clustering algorithm that partitions the data based on their density $[67,77]$. This clustering technique has been shown to perform well when performed in combination with UMAP dimension reduction [78] and it has been tested with several dataset types, including genetic data, grouping genes into the correct known classes [79]. Thus, we implemented HDBSCAN on the data obtained from UMAP analysis, which represents the abundance data in a reduced space of two dimensions. HDBSCAN analysis not only classifies OTUs/ASVs as belonging to a cluster but also as noise (defined as any point that was not selected in any of the clusters) and outliers (detected with the GLOSH outlier detection algorithm, which works with local outliers).

When running UMAP we set as default a minimum distance of 0.1 , number of components of 2, a Helligner output metric and number of neighbors of 15. In the case of HDBSCAN, the default parameters when running MicNet are Bray-Curtis metric, minimum cluster size of 15 , minimum sample size of 5 and quantile limit of 0.9 for outlier detection. For our different datasets, the number of neighbors (UMAP), and minimum cluster/sample size (HDBSCAN) parameters were set 
depending on the input microbiome dataset. We used Bray-Curtis dissimilarity as the distance metric for UMAP, as it is a standard metric for biological datasets. In the web dashboard, UMAP and HDBSCAN parameters can be modified by the user to visualize the results according to each set of microbiome data.

\section{New Implementation of SparCC}

To obtain the correlation matrix from relative abundance data, we implemented a modified version of the SparCC algorithm, a robust approach to discard spurious correlations when dealing with compositional data $[17,22]$. Although the original SparCC algorithm was not altered, several modifications were made to improve and scale the SparCC estimation matrix. We made three main changes to the original code; first, the code changed from Python version 2.7 to 3.9. Second, we use Numba and Dask in some parts of the matrix processes, namely functions or operations, with two main improvements: parallelization of operations and scalability in the size of the estimated matrices. Finally, the original SparCC version stores each estimation step in RAM, as arrays in NumPy. Although storing in RAM is efficient for small data sets, with large data the required memory increases rapidly depending on the interaction numbers and the size of the dataset. Thus, to overcome this problem, we store each estimation step on disk as hdf5 binary format. These changes made it possible to calculate the SparCC estimates with good performance in easily accessible computing resources. SparCC p-value test on the inferred correlation was not modified, it was calculated with a Monte-Carlo simulation (with default $n=50$ ) as done by Friedman \& Alm (2012) [17] and the default value is to calculate one-sided p-values, although this can be modified by the user.

To set SparCC parameter values, we perform a parameter search in our more complex study case from the communities reported by Espinosa-Asuar et al. (2021) [80]. We performed an independent parameter sweep on each parameter, varying the exclusion threshold from 0 to 1 in steps of 0.1 , the number of iterations from 10 to 100 in steps of 10 and the exclusion number from 10 to 100 in steps of 10 . For each parameter value, we calculated the number of correlations 

material). The final parameter values used in the databases presented here were: 50 iterations, an exclusion number of 10 , exclusion threshold of 0.10 and 100 simulations for $p$-value calculation. 210 However, this can be modified by the user both in the dashboard and when running the code from 211 the github repository.

\section{Network Analyses}

214 Network analyses were performed to characterize both the overall structure and the local interactions of the microbial co-occurrence network, in which each OTU/ASV is represented as a node and the correlations found by SparCC as undirected weighted edges, such that an edge

217 between two nodes implies a relationship between the two corresponding OTUs/ASVs. Given that most network analyses can only handle positive interactions, we normalized the SparCC correlation matrix from -1 to 1 to a range from 0 to 1 , except for the structural balance analysis which directly uses the positive and negative correlation values. All available network analyses in the MicNet toolbox are described as follows.

\section{Network Topology Comparison}

224 Networks have several large-scale structural measurements to characterize their topology. For this purpose, MicNet calculates the following structural metrics: 1) network density, using networkx function nx.density, 2) average degree, calculated as the mean of all nodes degree using numpy mean function, 3) degree standard deviation, using numpy std function, 4) ratio of positive-negative relationships calculated simply as the number of positively weighted edges divided by the number of negatively weighted edges,

5) average shortest path length using nx.average_shortest_path_length, 6) clustering coefficient nx.average_clustering function, 7) modularity, calculated with function nx.modularity, using as network modules those obtained with 
the nx.greedy_modularity_comminuties algorithm, and 8) the diameter, which was calculated using nx.diameter function. Finally, we have added a custom function that calculates a small-world (SW) index as suggested by Humphries \& Gurney (2008) [55]. The SW index is calculated as:

$$
S W=\frac{c c / c c_{\text {rand }}}{l / l_{\text {rand }}}
$$

Where $l$ and $c c$ are the average shortest path length and clustering coefficient of the experimental co-occurrence matrix, respectively. Analogously, $l_{\text {rand }}$ and $c c_{\text {rand }}$ are the average shortest path length and clustering coefficient, accordingly, of a comparable random network with the same number of nodes and density. The random network was built using the function nx.erdos_renyi_graph. This is done several times, with default $n=50$, and the mean value of SW 241 is returned. under the assumption that the underlying topology is: 1) a random Erdos-Renyi network [81] built using function nx.erdos_renyi_graph, 2) a small world Watts-Strogatz network [82] built using nx.watts_strogatz_graph function, or 3) a scale-free Barabási-Albert network [83] built using nx.barabasi_albert_graph function. A short description of these canonical topologies can be found in Fig 2. This allows the comparison of the query data against the different topologies. These simulated networks are built with the same number of nodes, density and average degree as the experimental data, and correlations are drawn from a uniform distribution from -1 to 1 . Finally, the simulated networks are made symmetrical to be comparable with the SparCC output correlation matrix.

Fig 2. Description of three canonical network topologies. Random, scale-free, and small-world topologies have different network properties. A brief description along with their type of degree distribution in shown. 

we have included in the MicNet toolbox a function that plots the Complementary Cumulative Distribution Function (CCDF) of the degrees of the given network and compares it with the CCDF of a simulated comparable random, scale-free and small-word network on a log-log scale. To calculate the CCDF we first divide the range of the degrees into bins; for each bin we obtain its probability as frequency/total. We used this discrete definition of the Probability Density Function (PDF) to calculate the Cumulative Density function (CDF) as the cumulative sum of the PDF:

where $x_{k}$ is the PDF of each bin $k$ previously defined, such that the CCDF is calculated as:

$$
C C D F=1-C D F
$$

We used CCDF since it has been suggested as an easier way to visualize the difference between degree distributions $[84,85]$.

271 To analyze subnetworks, we used two ways of dividing the network into subunits: 1) We used

272 Louvain method to detect communities in networks (using python-louvain library [86]), and 2) we 273 used the clusters found with clustering algorithm HDBSCAN. Each subnetwork's nodes and edges 274 were isolated as a subnetwork using function nx.subgraph, and then for each we obtained the 275 following metrics (also used for network topology analysis): total number of nodes, total number of relationships, density, average degree, and clustering coefficient. Finally, we characterize the

277 diversity of each subnetwork by looking at the total number of different taxa present in each 278 subnetwork at the phylum level. 


\section{Percolation Analysis}

281 Depending on the network structure, networks can be more or less robust to disruptions. Networks are usually formed by a giant component, which includes between $50 \%$ and $90 \%$ of the nodes. The formation and dissolution of this giant component is called percolation transition in network theory [87]. The percolation approach consists of removing nodes and their corresponding edges and analyzing how much the network's properties are disrupted [88]. The percolation simulation implemented in MicNet consists of $n$ iterations; in each iteration a percentage of the nodes (with default value of 0.1 , but this can be specified by the user) is removed along with all of their edges. After removing the nodes and corresponding edges, the following metrics are calculated for the remaining network: density, average degree, number of connected components (this last one calculated using the nx.connected_components function), size of giant component, fraction of nodes belonging to the giant component, the communities found by the python-louvain algorithm and the network modularity. We implemented several percolation approaches: 1) random percolation, in which nodes are removed randomly; 2) centrality percolation, in which nodes are removed by centrality (whether degree, closeness or betweenness centrality), higher values first; and 3) group percolation, where groups of nodes are removed according to a grouping variable provided, such as taxonomic groups or HDBSCAN groups. Consequently, network robustness to different types of disruptions could be assessed by looking at changes in different network metrics.

\section{Structural Balance Analysis}

300 Structural balance analysis finds all triangle motifs in the network, that is, nodes that are interacting in triads, and then classifies them as balanced based on the simple analogy that "my friend's friend is my friend" and "my friend's enemy is my enemy" $[89,90]$. This leads to classifying triads of interactions as balanced if they meet this criterion, or as imbalanced otherwise Fig 3. A network is considered to be balanced if most triads found in it are balanced. To calculate structural balance, we found all triads in the network using function nx.cycle_basis, and keeping only the cycles of length three. Then, we classified the found triangle motifs into balanced or imbalanced, depending 
on their mutual correlations. The output of the analysis is a percentage of balanced and imbalanced triangles with respect to all triangles found, and the exact percentage for each of the four types of triangles displayed in Fig 3.

311 Fig 3. Classification of triad motifs according to structural balance theory. Balanced or imbalanced criterion is assigned according to their mutual correlations.

313

\section{Potential Key Taxa Analysis}

315 Four different centrality measures were implemented to characterize each node (OTU/ASV): degree centrality using function nx.degree_centrality, betweenness centrality using function

317 nx.betweenness_centrality, closeness centrality using function nx.closeness_centrality and 318 PageRank using function nx.pagerank. When running the code, a dataset is returned with each centrality metric for each node.

\section{Dashboard Interface}

In addition to the freely accessible source code at the github repository, we have also developed a web dashboard at http://micnetapplb-1212130533.us-east-1.elb.amazonaws.com that can be used to run most of the analyses presented here. The dashboard consists of three main parts: 1) UMAP and HDBSCAN, 2) SparCC and 3) Network analyses. In the first component, the raw abundance data should be input (see Input Data section), and several parameters, as well as normalizations, for UMAP and HDBSCAN can be modified as desired. This first component returns an interactive visualization of the UMAP plot along with HDBSCAN clusters identified by color. Both the resulting plot and the file detailing cluster belonging can be downloaded from the dashboard. this section, abundance data should be input, and parameters can also be adjusted by the user. 
component includes the post-processing analyses of the co-occurrence network. Thus, the input of this section should be the matrix obtained from the previously defined SparCC component or any square correlation matrix, and the UMAP/HDBSCAN output file. When run, this section allows the user to obtain the large-scale metrics of the network, the structural balance percentages, descriptions of the communities found, and two network graphs where the size of the node indicates degree centrality, green edges indicate positive relationships between two nodes, whereas red edges indicate negative ones. Finally, in the graph named HDBSCAN the color of the nodes refers to the HDBSCAN cluster they belong to; whereas in the graph named Community the colors indicate the color of the community they belong to, based on Louvain clustering algorithm. When the network of interest consists of less than 500 nodes an interactive visualization plot is deployed, but for larger networks a static plot is returned, given limited computational resources. comparison, all the functions necessary to run them are in the github repository and can be used locally by users when downloading the toolbox. It should be noted that, if more computational resources are needed, the dashboard itself can be run after downloading the MicNet toolbox from github, creating the conda environment and deploying it with streamlit as suggested in the readme file.

\section{Validations}

\section{Modified SparCC and Topology Validations}

We performed two validations with simulated communities. We validated the new version of SparCC on the dataset provided by Friedman \& Alm (2012) [17], which consists of 50 OTUs in 200 samples drawn from a multinomial log-normal distribution. For this, we compared the real correlations to the estimations performed by SparCC, and then we calculated the RMSE (Root 
with 100 nodes each, and all of them with approximately the same density of $\rho=0.3$ and an average degree of 30 . Correlation matrices were simulated with three algorithms previously described to obtain: 1) a random network, 2) a scale-free network, and 3) a small-world network. The interaction magnitudes were simulated from a uniform distribution (range from -1 to 1 ). We calculated the different network metrics on each of these network topologies and used a bootstrap procedure to calculate the distribution of each metric given a certain topology. Finally, we also compare topologies using their degree CCDF, as described in a previous section.

\section{Biological Validation: Kombucha Consortium}

To further authenticate MicNet Toolbox's approach to analyze microbial co-occurrence networks, we needed to see if biological interactions previously described by experimental work could be replicated in the network. For this, we make use of the kombucha dataset described in Arıkan et al. (2020) [91]. Test data was downloaded from the European Nucleotide Archive (ENA) at EMBL$\mathrm{EBI}$ under the

accession

numbers

ERP104502

378 (https://www.ebi.ac.uk/ena/browser/view/ERP024546). The raw 16S amplicon reads were filtered, 379 processed and annotated with QIIME 2 [92] and DADA2 [93]. Abundance and taxonomy for each ASV cluster was acquired. The obtained abundance table with all samples was filtered, as singleton and unique counts were removed from the data, as suggested by Berry \& Widder (2014) [25]. Filtering unique and singletons resulted in 48 ASVs. For the visualization module, UMAP parameters were set as follows: number of neighbors of 5 , minimum distance of 0.10 , number of components of 2 and an Eucledian metric. In the case of HDBSCAN the parameters were: minimum cluster size of 5 , minimum sample size of 3 and Bray-Curtis metric. Network construction and network analyses were performed as described in previous sections. The raw data from the kombucha database is in the github repository so that the main results can be replicated and the user could interact with them in the dashboard. 


\section{Case Study: Archean Domes}

391 A 16S amplicon dataset was provided from a highly diverse microbial community named Archean

392 Domes. This dataset comes from a microbial mat located in the Cuatro Ciénegas Basin (CCB),

393 Coahuila, Mexico (coordinates $26^{\circ} 49^{\prime} 41.7^{\prime \prime} \mathrm{N}, 102^{\circ} 01^{\prime} 28.7^{\prime \prime} \mathrm{W}$ ). The sampling used in this case

394 study, which consists of ten samples along a $1.5 \mathrm{~m}$ transect, represents a natural community with 395 more than 6,000 ASVs [80]. Compositional data was acquired as raw reads form $16 \mathrm{~S}$ amplicon 396 sequencing. Reads were filtered and processed for clustering and taxonomic annotation in QIIME 3972 platform, as shown by the authors [80]. Singletons and unique counts were subsequently filtered as suggested, and consequently, 2,600 ASVs remained [25]. The ASV abundance matrices along with a taxonomic annotation for these sequences were used as input for the MicNet toolbox. For 400 the visualization module, UMAP and HDBSCAN parameters were set as follows: number of neighbors of 15 , minimum cluster size of 15 , and minimum sample size of 5 . Network construction and analyses were implemented as described in previous sections.

\section{Results}

\section{Validations}

\section{The Enhanced SparCC}

406 To validate that the modifications performed to SparCC did not affect its performance, we ran our 407 version of SparCC on the dataset provided by Friedman \& Alm (2012) [17]. We compared our 408 estimated correlation with their true basis correlation (Fig 4A-B). We found an overall RMSE of 4090.08 , and a consistent value of RMSE when estimating small and large correlation values from the 410 simulated samples (Fig 4C). Thus, although the original pipeline of SparCC was not modified, by 411 implementing several techniques that parallelized different parts of the code, our implementation 
412 of SparCC can now be used for large databases in a reasonable amount of time with relatively

413 small RMSE.

414

415 Fig 4. SparCC validation. The modified version of SparCC was validated using the database 416 provided by Friedman \& AIm (2012) [17]. A. Comparison of the estimated correlation with the real 417 correlation. B. Histogram of the estimation error produced with the new SparCC version. C. RMSE 418 across different absolute values of correlations, the overall RMSE error was 0.08 shown in the 419 dashed red line.

\section{Discerning between Network Topologies}

One prevailing question when analyzing microbial communities is whether these are randomly assembled or whether they follow certain non-random structures $[31,46,94]$. In order to validate that large-scale metrics can be used to differentiate between topologies, we began by creating three simulated networks with known random, small world or scale-free topology. All three networks were of 100 nodes each, and all three were made comparable in terms of density and average degree. Then, for each network topology we calculated the following large-scale structure metrics: clustering coefficient, average shortest path length, diameter, modularity, small-world index and degree standard deviation (Table S1: Supplementary material). For each topology, we simulated 300 networks and obtained the metrics mentioned above. This allowed us to obtain a distribution of each metric with different underlying topologies.

Results are shown in Fig 5, where we can see for each metric three distributions for random, scale-free and small-world networks. It can be appreciated that not all metrics are good to distinguish between topologies. In particular, modularity seems to be good to detect small-world topologies which display a higher modularity than that expected with random or scale-free topologies, but it does not distinguish between random and scale-free networks. Similarly, the network diameter is not a good indicator of topology, in fact, for scale-free and small-world networks, the diameter value in all 300 simulations was always 3 , so we were only able to plot a 
density distribution for random networks. In contrast, clustering coefficient, average path length, degree standard deviation and the small-word index seem to discriminate well between topologies.

Fig 5. Bootstrap distribution of large-scale metrics according to random, scale-free and small-word topology. Bootstrap distributions for random (red), scale-free (blue) and small-world lines represent the values for each topology shown in Table S1: Supplementary material. shows the histogram of each simulated network topology and the CCDF with a regression line fitted. We can see that the histogram of the scale-free network is clearly different from the other two topologies. Furthermore, the CCDF of the random network had the worst fit, with an explained variance of $r 2=0.66$, whereas the small world and scale-free CCDF show an almost perfect linear relationship, with explained variance of 0.89 and 0.88 respectively, as would be expected given their power-law distribution. Thus, degree distributions can also be used to give us hints of the underlying topology.

Fig 6. Degree distribution and CCDF for random, scale-free and small-world networks. Another way to differentiate between topologies is with their degree distribution, with CCDF allowing a clearer picture of whether it follows a power-law distribution, characteristic of small- world and scale-free networks. We show the histogram and CCDF with linear regression for an A. random network, B. scale-free network and C. small-world network. CCDF plots are on a log-log scale.

To demonstrate how MicNet tools could infer ecological associations, we used a kombucha data 
consortium of bacteria and yeast, which grow as biofilm due to cellulose production from acetic acid bacteria $(A A B)[91,95]$, but also develops as a liquid consortium. This consortium has been suggested as a convenient tractable system, whose general cooperative and antagonistic multispecies interactions have been previously described [95-97]. After filtering singletons and unique taxa from the raw data, only 48 ASVs remained in the analysis, corresponding to five annotated bacterial taxa and three fungal taxa.

As MicNet pipeline suggests, first, the community was visualized and analyzed with UMAP and HDBSCAN to uncover global patterns and noise taxa. Clusters from HDBSCAN showed one main group containing almost all ASV (34 of 48), a small group with 12 ASV and 2 ASV classified as noise shown in purple (Fig 7C). This could refer to a close-interacting community where highly stratified interactions are not common. Since the kombucha community has similar compositions in both homogeneous liquid and biofilms [91], physical closeness between all organisms is expected; this was reflected in the formation of one main group with the HDBSCAN algorithm. We then obtained the co-occurrence matrix of the kombucha samples using our modified version of SparCC. Table S2: Supplementary material shows the main metrics of the kombucha network and Fig $\mathbf{7 A}, \mathbf{B}$ show the resulting co-occurrence network.

Fig 7. Kombucha microbial network. A. SparCC co-occurrence network, where colors indicate Louvain groups. B. SparCC co-occurrence network, where color indicates the resulting HDBSCAN clusters. In purple is shown the group depicted as noise. C. UMAP and HDBSCAN results show one main group and a smaller one of 12 ASVs.

The resulting co-occurrence network was indeed a relatively connected one, with a connectance of 0.23 . This is reflected in the high average degree, which indicates that each ASV is related on average with around $10 \mathrm{ASVs}$ out of the 48 that are present in the community. Highly connected networks could point to a more homogenous environments, including liquid consortiums and slightly stratified biofilms in which kombucha develops, as opposed to more stratified environments, such as soil and microbial mat communities. The kombucha consortium is the result 
of a metabolic interplay between its microbial consortium, and though cheaters and antagonistic relationships are known [98], more cooperative relationships have been reported $[95,96]$. In fact, the network did show slightly more positive (55\%) than negative relationships (45\%), and a major contribution due to mutualist or commensalist interactions is expected.

We perform a topology comparison analysis to explore if the kombucha consortium fits within three canonical networks. Based on metrics bootstrapping from comparable networks, the kombucha network's degree standard deviation, average path length, SW index suggest some degree of small-worldness [55] (Fig S2: Supplementary material). Additionally, comparison of

502 kombucha CCDF with these simulated networks' CCDF suggest that the degree distribution of kombucha is not following particularly any specific canonical topology, although it appears to fit best with a random network (Fig S2: Supplementary material). These results are consistent, as kombucha medium is a non-stratified environment when it develops as liquid consortium, explaining the random properties, but still a stratified microbial consortium when developed as biofilm, explaining the scale-free properties. Thus, kombucha community plausibly shows properties in which microorganisms are adapted to biologically interact with each other in both homogeneous or heterogeneous (to some degree) structures.

Although some biological interactions in the kombucha consortium still need to be confirmed, the global interplay between AAB and yeasts is well-known. In kombucha fermentation, yeast produce invertase which cleave sucrose into glucose and fructose, and further use fructose

513 to produce ethanol. Ethanol is a noxious compound for the consortium. Hence, as a mechanism to 514 regulate ethanol concentrations in the media, $A A B$ transforms glucose and ethanol into gluconic 515 and acetic acid, respectively, exhibiting a straightforward case of syntrophy [91,95]. This biological 516 interplay was depicted in the ASV classified as Zygosaccharomyces baillii, the most abundant 517 yeast in the sample, and the ASV with the greatest number of interactions (Table S3: 518 Supplementary material). Second to Z. bailii, an ASV corresponding to Komagataeibacter europaeus (an $A A B)$ appears to be a key central taxa based on each centrality metric. According 520 to the inferred correlations by the enhanced SparCC (correlation matrices at the genus and species 521 level are provided in the Supplementary material as .pkl files), the $Z$. bailii correlated to every other 
522 ASVs, and mainly positively co-occurring with Komagataeibacter (0.1812), and negatively

523 correlating with Cortinarius (-0.2081). Actually, for the species within the Komagataeibacter show

524 the highest positively mean correlation with Z. bailii, particularly Komagataeibacter europeus

525 (0.5397), reflecting the cooperative interplay between yeast and AAB. Additionally,

526 Komagataeibacter, an AAB genus, produces acetic acid which inhibits growth of other species,

527 except for Z. bailii [91,99]. More specifically, it has been reported that $K$. rhaeticus is one of the main producers of acetic acid compared to other microbial species [100]. As expected, Komagataeibacter genus shows negative interactions against some of other species different from their own genus, such as Variovorax that is negatively correlated, which might be explained by with its growth-inhibiting capability. clustering analysis, where we found that communities were appreciably grouped per species, according to the Louvain method (Fig S3: Supplementary material). Clustering resulting from phylogenetic relatedness is common in microbial data, and it may reflect niche overlapping to some degree [4,31]. From the 5 communities predicted with the Louvain method, one of them is considered as noise as it consists of just 1 ASV. HDBSCAN group composition is variable, as most ASV belong to just one group (Fig S4: Supplementary material). Nonetheless, the smaller group with 12 ASV's from HDBSCAN is particularly interesting, as it includes most of $K$. europaeus and the Z. bailii ASV, probably depicting the core syntrophic interactions. This potential core group is

542 similarly shown as a Louvain method group. Main metrics for each group of the community analysis

543 (via Louvain or HDBSCAN groups) are reported in Table S4 and S5: Supplementary material. player in the metabolic interplay with $A A B, A A B$ are not fully dependent on them for substrates, characterizing their interaction as some class of non-strict parasitism [97]. In the co-occurrence network, we found evidence that Z. bailii was indeed considered a key organism given its high

548 centrality metrics, but in the percolation analysis where nodes were removed by degree centrality

549 (beign Z. bailii), there was not a breakdown of the network (nor in the network density, the average 
550 degree, the number of components or the number of communities, as shown in Table S6:

551 Supplementary material, along with other percolation analyses performed). In contrast,

552 percolation analysis where the nodes are removed depending on their genus exhibit a network

553 breakup of several components when most of Komagataeibacter nodes were removed. Even by

554 removing six Komagataeibacter nodes, the network is disrupted into three components, further

555 supporting the relevance of Komagataeibacter in the kombucha network. Percolation analysis and centrality metrics are consistent in positioning the Komagataeibacter as a crucial genus to the community, and this can be biologically understood due to 1) their independence (to some degree) from yeast to thrive , 2) their cellulose production capability (as a mechanism for protection and resource storage [95]), and 3) as regulators on ethanol concentration.

\section{Case Study: Archean Domes Microbial Mats}

562 To further evaluate the performance of MicNet as a high-throughput toolbox capable of analyzing 563 a highly diverse and complex environment, we tested it on a compositional dataset of ten samples 564 from a microbial mat in Cuatro Ciénegas, Mexico, in the Chihuahuan Desert [80]. This microbial mat, The Archean Domes, thrives in a fluctuating hypersaline pond which has been previously described as hyperdiverse $[80,101]$. Like every microbial mat, it is a stratified community with an intricate metabolic interplay between their organisms [102]. Espinosa-Asuar et al. (2021) provided us their microbial data set of 6,063 ASVs, as they have reported. To begin the pipeline, the abundance matrix for all ASV was filtered to exclude low abundance and unique ASVs, remaining 2,600 amplicon sequence variants for the analysis. in each group (Fig $\mathbf{8 C}$ ). With this approach, the HDBSCAN algorithm allowed us to categorize organisms as noise or outliers, as 553 ASV did not group with any cluster and were categorized as noise, and 260 behaved as outliers. 
577 Fig 8. Archean Domes biological network. A. SparCC co-occurrence network, where different

578 colors indicate Louvain groups. B. SparCC co-occurrence network, where color indicates the 579 resulting HDBSCAN clusters. C. UMAP and HDBSCAN results show 50 clusters and several ASV 580 classified as noise and outliers

581

582

583

584

585

586

587

588

589

590

591

592

593

594

595

596

597

598

599

600

601

602

603

Afterwards, we computed the correlation matrix with the modified version of SparCC. Main large-scale characteristics of the network are shown in Table S7: Supplementary material. With the 2,600 ASV we recreated a network with 463,609 interactions with all of them aggregated in a big but sparsely connected network (density $=0.137)(\mathbf{F i g} \mathbf{8 A}, \mathbf{B})$. This value correlates with the interaction density of an antagonist network between 37 Gammaproteobacteria strains isolated from water samples of Churince (reported density $=0.14$ ), a lake situated also in the CCB [103], further suggesting the fact that microbial mats' networks are often sparse $[1,31]$.

Network topology comparison for the Archean Domes network display how high-complexity systems do not fit greatly simplified theoretical models. This was shown in the metrics bootstrapping from simulated comparable networks, where most Archean Domes metrics fall in between a scalefree and small-world network distributions (Fig S5: Supplementary material). Particularly, degree standard deviation, modularity, SW index, and clustering coefficient suggest that Archean Domes possess intermediate properties between scale-free and small-world networks. On the other hand, the average path length of 1.86 is typical of a scale-free network. These results show that this community is not randomly assembled, and that as expected from microbial data, the network is likely an intermediate between a scale-free and a small-world network $[31,55]$.

Potential key taxa analysis based on node centrality was performed for this high-complexity network. An ASV corresponding to a bacterium from the order MSBL9, class Phycisphaerae, phylum Planctomycetes, exhibit the highest centrality values, regardless of the centrality measure employed (Table S8: Supplementary material). This bacterial class has been previously described in hypersaline microbial mats as anoxic, fermenting, halotolerant and halophilic microorganisms $[104,105]$. Looking at other top centrality nodes, most of them were associated to 
unassigned sequences, except for a node member of the class Parcubacteria and another one from the genus Imperialibacter (Table S8: Supplementary material). Moreover, unknown taxa as central nodes further suggest the relevance of "microbial dark matter" in ecosystems, and particularly, in hypersaline environments like the one studied here [106]. matrices at the phylum and species level are provided in the Supplementary material as .pkl files).

610 From the 463,610 total relationships in the network, we only highlight some of them which might

611 be insightful. At the phylum level, ASVs from Proteobacteria, the most abundant phylum in the 612 sample, have positive mean correlations with most of the phyla, except Nanoarchaeota,

613 Dependentiae and other unassigned prokaryotes. Cyanobacteria, a key phylum in microbial mats, 614 on the other hand face more negative mean correlations with other phyla, including Synergistetes, 615 Acetothermia and Chloroflexi. These negative correlations prospectively originate from overall antagonistic interactions or different niche requirements (such as wavelength, carbon metabolism

617 or temperature adaptation for potential chloroflexi-cyanobacteria associations [107]). Lower 618 taxonomic associations could be inspected. For example, the most central hub taxa (unassigned 619 MSBL9, class Phycisphaerae) positively co-occurs the most (0.5903) with a bacterium from the genus Dehalobium, while negatively co-occurs the most with a deltaproteobacteria from the Syntrophobacteraceae (-0.6177).

Although most ecological associations (particularly biological interactions) are analyzed by pairs, species also associate and interact involving more than two organisms, which are vital for ecosystem diversity [108]. Triad motif identification and structural balance theory attempts to address this issue in microbial networks. For the Archean Domes network, we identify that most of the triads are balanced, with a highly balanced triad fraction of 0.9995 . One property of structural balanced networks is group division, wherein all intergroup ties are negative, and all intragroup ties are positive [109]. Potentially applied to microbial ecology, we suggest that structural balance could reflect niche differentiation, in conjunction with other network metrics and properties that have been previously described as useful $[1,4,110]$. 
Furthermore, we carried out a community analysis to inspect subnetwork properties. Our two clustering methods, Louvain and HDBSCAN groups, display contrasting results, which could

633 reflect different ecological structures. Louvain grouping algorithm resulted in 7 network 634 communities with mean total nodes of 371.43 and mean density of 0.4269 , while HDBSCAN showed 50 clusters with mean total nodes of 40.94 and mean density of 0.73 . Main metrics for each group, for each grouping algorithm, are shown in Table S9 and S10: Supplementary material. Phyla composition within most groups (either Louvain or HDBSCAN) is highly diverse, which could mirror spatial structures or compartments at different scales [3,56], which is consistent with the stratified structure of microbial mats [102].

High-diversity communities are commonly associated with an overall stable state. While the

641 high number of ASV in Archean Domes probably reflects a highly diverse system, novel methods

642 to assess ecosystem stability have been suggested. In microbiomes, positive relationships alone

643 are prone to destabilize microbial networks, as they can create highly dependent and vulnerable

644 feedback loops $[3,111]$. Archean Domes co-occurrence network show a negative:positive ratio of

6450.94 , thus, positive and negative relationships are evenly present, suggesting an ecologically

646 resilient, resistant and stablished community. Within this scheme, negative relationships in the

647 community might be originating from antagonistic competitive taxa, lower abundance of facilitative

648 taxa (mutualists), divergent niche requirements, or a combination of all of them $[1,3]$. Modularity

649 further bolster the stability hypothesis within the microbial mat. Modular groups (or clusters), whether product of biological interactions or habitat preference, plausibly aid in the stability of the system, as fewer links between groups likely ameliorate the spread of local perturbations to other

652 groups. Modularity in Archean Domes shows a high value of 0.34 , higher than the kombucha network and other published biological networks [3]. Low average path length (1.86 in Archean Domes network) likely function as a measure of response capacity to disturbances, hinting about the ecological resilience capabilities of this microbial mat [4,31]. Finally, Percolation analysis delves deeper into community stability. We performed random, centrality and phylum percolation

657 simulations, from which the resulting metrics are shown in Table S11: Supplementary material.

658 While none of the node removals induce the breakup of the giant component, the total number of 
communities and overall modularity do perceive the effects of network perturbations. With this in

660 mind, some degree of ecological robustness could be reflected by the impact of percolation simulations to the co-occurrence network.

662

\section{Discussion}

664

665

666

667

668

669

670

671

672

673

674

675

676

677

678

679

MicNet toolbox has shown to be a promising pipeline. Our new implementation of the SparCC algorithm allows larger datasets to be processed without overflowing the RAM in a simple way in our web dashboard. Furthermore, UMAP and HDBSCAN, relatively new dimension reduction and clustering techniques that are promising in microbial ecology studies since, as suggested in this work, they are useful methods to identify metabolic groups, niche overlapping, or subcommunities,. Finally, given the potential of processing co-occurrence networks with a graph theory approach, we have included several network analyses, both new and commonly used, to further describe and understand the resulting networks from SparCC.

One important aspect to have in mind when using the MicNet toolbox is sample size. UMAP and HDBSCAN are known to be quite sensitive to the size of the database used. With a very small dataset (less than 50 OTUs/ASVs) we recommend being cautious at the interpretation level. Dalmaijer et al. (2020) [78] have suggested that for optimal use of UMAP and HDBSCAN, it is recommended to have around 20-30 data points per expected cluster or subgroup. Furthermore, the choice of parameters for these two techniques should be done with careful consideration, in particular the number of nearest neighbors and minimum distance [65]. We hope that the interactive dashboard will help in this aspect, since parameters can be modified in a simple way.

In terms of network topology, not all large-scale metrics of a network should be used to discern between topologies. As we show previously, some metrics, such as diameter, have no use to discern topologies, whereas others, like the small-word index provide useful information [55]. Moreover, it is unlikely that any biological system follows exactly a single topology, as shown by our case study: Archean Domes, the hyperdiverse microbial mats in CCB. However, we believe 
that knowing whether a network tends more towards a random, scale-free or small-world could give insightful pointers about its general behavior. For example, a small-world model (created with the Watts-Strogatz algorithm) suggests that the network will have short path lengths, because it is formed by highly connected clusters, which are weakly connected among each other; whereas a scale-free model (created with the Barabasi-Albert algorithm) suggests that networks will have short average path lengths as well, as a consequence that certain nodes that have very high degree and can act as hubs; in both cases the average distance between nodes would be expected to be small but for different reasons, which could give us insight of key biological network properties [85]. Microbial communities in the light of complexity has showed, once again, the potential in drawing biological conclusions from networks. Nonetheless, the biological interpretation of UMAP, HDBSCAN and network analysis should also be taken cautiously. As we mentioned before, the interpretation of the different metrics obtained is debatable, and there is a high diversity of interpretations and terms (see synonyms in Table 1) used for the same ecological concepts. As for today, researchers are encouraged to correlate network theory metrics to biological significance in an attempt to find fundamental metrics that could be useful to describe a particular biological phenomenon in whichever microbial system. With MicNet we suggest an analysis pipeline including visualization, co-occurrence network creation and postprocessing of the resulting network with graph theory analyses that could be used as a standard method for network analysis, offering an overview of a microbial community and enabling the comparison between different microbial systems. Potentially, this approach promises to aid in the search for biologically fundamental metrics.

Biological validation with a kombucha consortium was accomplished, as known local and

707 global behavior, including key taxa and interspecific biological interactions empirically confirmed elsewhere $[91,95]$, were reproduced by the proposed toolbox. Moreover, our high-complexity case study, Archean Domes, displays the scope and usefulness of MicNet toolbox by deconstructing microbial co-occurrence networks to manageable biological knowledge. Aware of current caveats 711 of the limitations microbial co-occurrence networks have $[1,26]$, we restate that this approach 712 should be taken as a roadmap for further research on the microbiome system, rather than a 
713 conclusive analysis. For example, directed studies to Phycisphaerae bacteria (and other central

714 taxonomic groups) can be performed, and consequently, assess the relevance of this taxon to the

715 whole community structure and functioning. Similarly, "microbial dark matter" characterization and

716 relevance could be further explored with the increasing technologies and databases [112]. Directed

717 co-culture experiments and other novel strategies such as microdroplets are fundamental for

718 biological interactions' validation [31], which could be applied to inferred correlations between taxa

719 of interest. Moreover, module aimed experiments, including synthetic microbial communities, are

720 tractable strategies that, although reducing complexity of the system, could be informative about

721 mid-scale structures crucial to the system's stability [113], especially if the experiments include

722 perturbations [114].

723 Given its potential usefulness, understanding both global and local patterns in microbial

724 communities may be a wise strategy to delve deeper into their currently unknown properties. With

725 the introduction of the MicNet toolbox, we hope that the research community will be able to

726 implement several existing and new analysis techniques in a straightforward manner to further keep

727 unravelling the intricate conundrums that microbiomes hold.

728

729

\section{Availability and Further Directions}

730 MicNet toolbox is available to the scientific community as an open source project at

731 https://github.com/Labevo/MicNetToolbox. In addition, we present an accompanying dashboard

732 which can also be freely visited at http://micnetapplb-1212130533.us-east-1.elb.amazonaws.com,

733 in which the files to build the kombucha network or any other compositional files the user inputs,

734 can be explored and visualized in an interactive way.

735

\section{Acknowledgments}


We thank Diego Nava for his contributions in the conceptualization and management of the project and Julian Trejo and Diana Fernandez Rosales for their contribution in the elaboration of the artwork. We thank Erika Aguirre for her technical support.

\section{Financial Disclosure Statement}

742 This research was supported by PhD scholarship 970341 granted by Consejo Nacional de Ciencia

\section{Competing interests}

The authors declare no competing financial interests.

\section{References}

1. Röttjers L, Faust K. From hairballs to hypotheses-biological insights from microbial

2. Dohlman AB, Shen $X$. Mapping the microbial interactome: Statistical and experimental approaches for microbiome network inference. Exp. Biol. Med. 2019;244:445.

3. Hernandez DJ, David AS, Menges ES, Searcy CA, Afkhami ME. Environmental stress destabilizes microbial networks. ISME J. 2021156 2021;15:1722-34.

4. Karimi B, Maron PA, Chemidlin-Prevost Boure N, Bernard N, Gilbert D, Ranjard L. Microbial diversity and ecological networks as indicators of environmental quality. Environ. Chem. Lett. 2017152 2017;15:265-81.

5. Poudel R, Jumpponen A, Schlatter DC, Paulitz TC, Gardener B., Kinkel LL, et al. Microbiome Networks: A Systems Framework for Identifying Candidate Microbial Assemblages for Disease Management. Phytopathology 2016;106:1083-96.

6. Xiaofei L, Kankan Z, Ran X, Yuanhui L, Jianming X, Bin M. Strengthening Insights in Microbial Ecological Networks from Theory to Applications. mSystems 2010;4:e00124-19.

7. Pearson K. Determination of the coefficient of correlation. Science (80-. ). 1909;30:23-5.

8. Spearman C. The Proof and Measurement of Association between Two Things. Am. J. Psychol. 1904;15:72.

9. Jaccard $P$. The distribution of the flora in the alpine zone. New Phytol. 1912;11:37-50.

10. Bray JR, Curtis JT. An Ordination of the Upland Forest Communities of Southern Wisconsin. Ecol. Monogr. 1957;27:325-49.

11. Ruan Q, Dutta D, Schwalbach MS, Steele JA, Fuhrman JA, Sun F. Local similarity analysis reveals unique associations among marine bacterioplankton species and environmental factors. Bioinformatics 2006;22:2532-8.

12. Xia LC, Ai D, Cram J, Fuhrman JA, Sun F. Efficient statistical significance approximation for local similarity analysis of high-throughput time series data. Bioinformatics 2013;29:230-7.

13. Reshef DN, Reshef YA, Finucane HK, Grossman SR, McVean G, Turnbaugh PJ, et al. 
14. Jizhong Z, Ye D, Feng L, Zhili H, Yunfeng Y, David R. Phylogenetic Molecular Ecological Network of Soil Microbial Communities in Response to Elevated CO2. MBio 2021;2:e00122-11.

15. Deng Y, Jiang Y-H, Yang Y, He Z, Luo F, Zhou J. Molecular ecological network analyses. BMC Bioinforma. 2012131 2012;13:1-20.

16. Aitchison J. The statistical analysis of compositional data. London; New York: Chapman and Hall; 1986.

17. Friedman J, Alm EJ. Inferring Correlation Networks from Genomic Survey Data. PLOS Comput. Biol. 2012;8:e1002687.

18. Faust K, Sathirapongsasuti JF, Izard J, Segata N, Gevers D, Raes J, et al. Microbial Cooccurrence Relationships in the Human Microbiome. PLOS Comput. Biol. 2012;8:e1002606.

19. Faust K, Raes J. CoNet app: inference of biological association networks using Cytoscape. F1000Research 2016;5:1519.

20. Succurro A, Ebenhöh O. Review and perspective on mathematical modeling of microbial ecosystems. Biochem. Soc. Trans. 2018;46:403.

21. Volterra V. Variazioni e fluttuazioni del numero d'individui in specie animali conviventi. 1926.

22. Weiss S, Van Treuren W, Lozupone C, Faust K, Friedman J, Deng Y, et al. Correlation detection strategies in microbial data sets vary widely in sensitivity and precision. ISME J. 2016107 2016;10:1669-81.

23. Shaw GT-W, Pao Y-Y, Wang D. MetaMIS: a metagenomic microbial interaction simulator based on microbial community profiles. BMC Bioinforma. 2016171 2016;17:1-12.

24. Fisher CK, Mehta P. Identifying Keystone Species in the Human Gut Microbiome from Metagenomic Timeseries Using Sparse Linear Regression. PLoS One 2014;9:e102451.

25. Berry D, Widder S. Deciphering microbial interactions and detecting keystone species with co-occurrence networks. Front. Microbiol. 2014;0:219.

26. Hirano $\mathrm{H}$, Takemoto $\mathrm{K}$. Difficulty in inferring microbial community structure based on cooccurrence network approaches. BMC Bioinforma. 2019201 2019;20:1-14.

27. Raman K. Structure of Networks. In: An Introduction to Computational Systems Biology: Systems-Level Modelling of Cellular Networks. Chapman and Hall/CRC.; 2021. page 5790.

28. Landi P, Minoarivelo HO, Brännström Å, Hui C, Dieckmann U. Complexity and stability of ecological networks: a review of the theory. Popul. Ecol. 2018604 2018;60:319-45.

29. Bouchez T, Blieux AL, Dequiedt S, Domaizon I, Dufresne A, Ferreira S, et al. Molecular microbiology methods for environmental diagnosis. Environ. Chem. Lett. 2016144 2016;14:423-41.

30. Loreau M, Naeem S, Inchausti P, Bengtsson J, Grime JP, Hector A, et al. Biodiversity and ecosystem functioning: Current knowledge and future challenges. Science (80-. ). 2001;294:804-8.

31. Faust K, Raes J. Microbial interactions: from networks to models. Nat. Rev. Microbiol. 2012108 2012;10:538-50.

32. Mandakovic D, Rojas C, Maldonado J, Latorre M, Travisany D, Delage E, et al. Structure and co-occurrence patterns in microbial communities under acute environmental stress reveal ecological factors fostering resilience. Sci. Reports 201881 2018;8:1-12.

33. Elmqvist T, Folke C, Nyström M, Peterson G, Bengtsson J, Walker B, et al. Response diversity, ecosystem change, and resilience. Front. Ecol. Environ. 2003;1:488-94.

34. Tylianakis JM, Laliberté E, Nielsen A, Bascompte J. Conservation of species interaction networks. Biol. Conserv. 2010;143:2270-9.

35. Zappelini C, Karimi B, Foulon J, Lacercat-Didier L, Maillard F, Valot B, et al. Diversity and complexity of microbial communities from a chlor-alkali tailings dump. Soil Biol. Biochem. 2015;90:101-10.

36. De Anda V, Zapata-Peñasco I, Blaz J, Poot-Hernández AC, Contreras-Moreira B, González-Laffitte M, et al. Understanding the Mechanisms Behind the Response to 
861

862

863

864

865

866

867

868

869

870

871

872

873

874

875

876

877

878

879

880

881

882

883

884

Environmental Perturbation in Microbial Mats: A Metagenomic-Network Based Approach. Front. Microbiol. 2018;0:2606.

37. Zhou H, Gao Y, Jia X, Wang M, Ding J, Cheng L, et al. Network analysis reveals the strengthening of microbial interaction in biological soil crust development in the Mu Us Sandy Land, northwestern China. Soil Biol. Biochem. 2020;144:107782.

38. Corel E, Lopez P, Méheust R, Bapteste E. Network-Thinking: Graphs to Analyze Microbial Complexity and Evolution. Trends Microbiol. 2016;24:224-37.

39. Connor N, Barberán A, Clauset A. Using null models to infer microbial co-occurrence networks. PLoS One 2017;12:e0176751.

40. Barroso-Bergadà D, Pauvert $C$, Vallance J, Delière L, Bohan DA, Buée $M$, et al. Microbial networks inferred from environmental DNA data for biomonitoring ecosystem change: Strengths and pitfalls. Mol. Ecol. Resour. 2021;21:762-80.

41. de Vries FT, Griffiths RI, Bailey M, Craig H, Girlanda M, Gweon HS, et al. Soil bacterial networks are less stable under drought than fungal networks. Nat. Commun. 201891 2018;9:1-12.

42. Dong $Y$, Gao J, Wu Q, Ai Y, Huang Y, Wei W, et al. Co-occurrence pattern and function prediction of bacterial community in Karst cave. BMC Microbiol. 2020201 2020;20:1-13.

43. Hannigan GD, Duhaime MB, Koutra D, Schloss PD. Biogeography and environmental conditions shape bacteriophage-bacteria networks across the human microbiome. PLoS Comput. Biol. 2018;14.

44. Liu Z, Wei H, Zhang J, Saleem M, He Y, Zhong J, et al. Higher Sensitivity of Soil Microbial Network Than Community Structure under Acid Rain. Microorg. 2021, Vol. 9, Page 118 2021;9:118.

45. Schmoldt A, Benthe HF, Haberland G. Digitoxin metabolism by rat liver microsomes. Biochem. Pharmacol. 1975;24:1639-41.

46. Layeghifard M, Hwang DM, Guttman DS. Disentangling Interactions in the Microbiome: A Network Perspective. Trends Microbiol. 2017;25:217.

47. Estrada-Peña A, Cabezas-Cruz A, Pollet T, Vayssier-Taussat M, Cosson J-F. High Throughput Sequencing and Network Analysis Disentangle the Microbial Communities of Ticks and Hosts Within and Between Ecosystems. Front. Cell. Infect. Microbiol. 2018;8:236.

48. Herren $\mathrm{CM}$, McMahon KD. Cohesion: a method for quantifying the connectivity of microbial communities. ISME J. 20171111 2017;11:2426-38.

49. Coyte KZ, Schluter J, Foster KR. The ecology of the microbiome: Networks, competition, and stability. Science (80-. ). 2015;350:663-6.

50. Suweis S, Grilli J, Maritan A. Disentangling the effect of hybrid interactions and of the constant effort hypothesis on ecological community stability. Oikos 2014;123:525-32.

51. Mougi A, Kondoh M. Diversity of interaction types and ecological community stability. Science (80-. ). 2012;337:349-51.

52. Zhou J, Deng Y, Luo F, He Z, Tu Q, Zhi X. Functional molecular ecological networks. MBio 2010;1.

53. Lupatini M, Suleiman AKA, Jacques RJS, Antoniolli ZI, de Siqueira Ferreira A, Kuramae $\mathrm{EE}$, et al. Network topology reveals high connectance levels and few key microbial genera within soils. Front. Environ. Sci. 2014;0:10.

54. Barberán A, Bates ST, Casamayor EO, Fierer N. Using network analysis to explore cooccurrence patterns in soil microbial communities. ISME J. 201262 2011;6:343-51.

55. Humphries MD, Gurney K. Network 'Small-World-Ness': A Quantitative Method for Determining Canonical Network Equivalence. PLoS One 2008;3:e0002051.

56. Cram JA, Xia LC, Needham DM, Sachdeva R, Sun F, Fuhrman JA. Cross-depth analysis of marine bacterial networks suggests downward propagation of temporal changes. ISME J. 2015912 2015;9:2573-86.

57. Zhang B, Zhang J, Liu Y, Shi P, Wei G. Co-occurrence patterns of soybean rhizosphere microbiome at a continental scale. Soil Biol. Biochem. 2018;118:178-86.

58. Dubin K, Callahan MK, Ren B, Khanin R, Viale A, Ling L, et al. Intestinal microbiome analyses identify melanoma patients at risk for checkpoint-blockade-induced colitis. Nat. 
Commun. 201671 2016;7:1-8.

59. McHardy IH, Goudarzi M, Tong M, Ruegger PM, Schwager E, Weger JR, et al. Integrative analysis of the microbiome and metabolome of the human intestinal mucosal surface reveals exquisite inter-relationships. Microbiome 201311 2013;1:1-19.

60. Guidi L, Chaffron S, Bittner L, Eveillard D, Larhlimi A, Roux S, et al. Plankton networks driving carbon export in the oligotrophic ocean. Nat. 20165327600 2016;532:465-70.

61. Saberi M, Khosrowabadi R, Khatibi A, Misic B, Jafari G. Topological impact of negative links on the stability of resting-state brain network. Sci. Reports 2021111 2021;11:1-14.

62. Ma Z (Sam), Ye D. Trios-promising in silico biomarkers for differentiating the effect of disease on the human microbiome network. Sci. Reports 2017 71 2017;7:1-9.

63. Srinivasan A. Local balancing influences global structure in social networks. Proc. Natl. Acad. Sci. 2011;108:1751-2.

64. Mclnnes L, Healy J, Saul N, Großberger L. UMAP: Uniform Manifold Approximation and Projection. J. Open Source Softw. 2018;3:861.

65. Diaz-Papkovich A, Anderson-Trocmé L, Ben-Eghan C, Gravel S. UMAP reveals cryptic population structure and phenotype heterogeneity in large genomic cohorts. PLOS Genet. 2019;15:e1008432.

66. Diaz-Papkovich A, Anderson-Trocmé L, Gravel S. A review of UMAP in population genetics. J. Hum. Genet. 2020661 2020;66:85-91.

67. Mclnnes L, Healy J, Astels S. hdbscan: Hierarchical density based clustering. J. Open Source Softw. 2017;2:205.

68. Van Rossum G, Drake FL. Python 3 Reference Manual. Scotts Valley, CA: CreateSpace; 2009.

69. McKinney W. Data Structures for Statistical Computing in Python. In: Proceedings of the 9th Python in Science Conference. SciPy; 2010. page 56-61.

70. The pandas development team. pandas-dev/pandas: Pandas 1.3.2. 2021;

71. Harris CR, Millman KJ, van der Walt SJ, Gommers R, Virtanen P, Cournapeau D, et al. Array programming with NumPy. Nat. 20205857825 2020;585:357-62.

72. Dask Development Team. Dask: Library for dynamic task scheduling. 2016;

73. Lam SK, Pitrou A, Seibert S. Numba: A Ilvm-based python jit compiler. In: Proceedings of the Second Workshop on the LLVM Compiler Infrastructure in HPC. 2015. page 1-6.

74. Collette A. Python and HDF5. O'Reilly; 2013.

75. Bokeh Development Team. Bokeh: Python library for interactive visualization. 2018;

76. Hagberg AA, Schult DA, Swart PJ. Exploring Network Structure, Dynamics, and Function using NetworkX. In: Varoquaux G, Vaught T, Millman J, editors. Proceedings of the 7th Python in Science Conference. Pasadena, CA USA: 2008. page 11-5.

77. Malzer C, Baum M. A Hybrid Approach To Hierarchical Density-based Cluster Selection. IEEE Int. Conf. Multisens. Fusion Integr. Intell. Syst. 2019;2020-September:223-8.

78. Dalmaijer ES, Nord CL, Astle DE. Statistical power for cluster analysis. 2020;

79. Campello RJGB, Moulavi D, Sander J. Density-Based Clustering Based on Hierarchical Density Estimates. Lect. Notes Comput. Sci. (including Subser. Lect. Notes Artif. Intell. Lect. Notes Bioinformatics) 2013;7819 LNAI:160-72.

80. Espinosa-Asuar L, Monroy C, Madrigal-Trejo D, Navarro M, Sánchez J, Muñoz J, et al. Ecological relevance of abundant and rare taxa in a high-diverse elastic hypersaline microbial mat, using a small-scale sampling. bioRxiv 2021;2021.03.04.433984.

81. Erdös P, Rényi A. On Random Graphs I. Publ. Math. Debrecen 1959;6:290.

82. Watts DJ, Strogatz SH. Collective dynamics of 'small-world' networks. Nat. 19983936684 1998;393:440-2.

83. Barabási A-L, Albert R. Emergence of Scaling in Random Networks. Science (80-. ). 1999;286:509-12.

84. Alizadeh M, Cioffi-Revilla C, Crooks A. Generating and analyzing spatial social networks. Comput. Math. Organ. Theory 2016233 2016;23:362-90.

85. Downey A. Think complexity : complexity science and computational modeling. Sebastopol, Calif.: O'Reilly; 2018.

86. Aynaud T. python-louvain x.y: Louvain algorithm for community detection. 2020; 
940 87. Newman MEJ. Networks : an introduction. Oxford [u.a.]: Oxford Univ. Press; 2010.

88. Barabási A-L, Pósfai M. Network science. 2016.

942

943

944

945

946

947

948

949

950

951

952

953

954

955

956

957

958

959

960

961

962

963

964

965

966

967

968

969

970

971

972

973

974

975

976

977

978

979

980

981

982

983

984

985

986

987

89. Aref S, Wilson MC. Balance and frustration in signed networks. J. Complex Networks 2019;7:163-89.

90. Estrada E. Rethinking structural balance in signed social networks. Discret. Appl. Math. 2019;268:70-90.

91. Arıkan M, Mitchell AL, Finn RD, Gürel F. Microbial composition of Kombucha determined using amplicon sequencing and shotgun metagenomics. J. Food Sci. 2020;85:455-64.

92. Bolyen E, Rideout JR, Dillon MR, Bokulich NA, Abnet CC, Al-Ghalith GA, et al.

Reproducible, interactive, scalable and extensible microbiome data science using QIIME 2. Nat. Biotechnol. 2019378 2019;37:852-7.

93. Callahan BJ, McMurdie PJ, Rosen MJ, Han AW, Johnson AJA, Holmes SP. DADA2: High resolution sample inference from Illumina amplicon data. Nat. Methods 2016;13:581.

94. Palla G, Derényi I, Farkas I, Vicsek T. Uncovering the overlapping community structure of complex networks in nature and society. Nat. 20054357043 2005;435:814-8.

95. May A, Narayanan S, Alcock J, Varsani A, Maley C, Aktipis A. Kombucha: a novel model system for cooperation and conflict in a complex multi-species microbial ecosystem. PeerJ 2019;7.

96. BE W, RJ D. Fermented foods as experimentally tractable microbial ecosystems. Cell 2015;161:49-55.

97. Tran T, Grandvalet C, Verdier F, Martin A, Alexandre H, Tourdot-Maréchal R. Microbial Dynamics between Yeasts and Acetic Acid Bacteria in Kombucha: Impacts on the Chemical Composition of the Beverage. Foods 2020;9.

98. Jayabalan R, Malbaša R V., Lončar ES, Vitas JS, Sathishkumar M. A Review on Kombucha Tea-Microbiology, Composition, Fermentation, Beneficial Effects, Toxicity, and Tea Fungus. Compr. Rev. Food Sci. Food Saf. 2014;13:538-50.

99. Dang TDT, Vermeulen A, Ragaert P, Devlieghere F. A peculiar stimulatory effect of acetic and lactic acid on growth and fermentative metabolism of Zygosaccharomyces bailii. Food Microbiol. 2009;26:320-7.

100. Yamada Y, Yukphan P, Vu HTL, Muramatsu Y, Ochaikul D, Tanasupawat S, et al. Description of Komagataeibacter gen. nov., with proposals of new combinations (Acetobacteraceae). J. Gen. Appl. Microbiol. 2012;58:397-404.

101. Medina-Chávez NO, De la Torre-Zavala S, Arreola-Triana AE, Souza V. Cuatro Ciénegas as an Archaean Astrobiology Park. Springer, Cham; 2020. page 219-28.

102. Prieto-Barajas CM, Valencia-Cantero E, Santoyo G. Microbial mat ecosystems: Structure types, functional diversity, and biotechnological application. Electron. J. Biotechnol. 2018;31:48-56.

103. Aguirre-von-Wobeser E, Soberón-Chávez G, Eguiarte LE, Ponce-Soto GY, VázquezRosas-Landa M, Souza V. Two-role model of an interaction network of free-living $Y$ proteobacteria from an oligotrophic environment. Environ. Microbiol. 2014;16:1366-77.

104. Spring S, Bunk B, Spröer C, Rohde M, Klenk H-P. Genome biology of a novel lineage of planctomycetes widespread in anoxic aquatic environments. Environ. Microbiol. 2018;20:2438-55.

105. Thomas F, Morris JT, Wigand C, Sievert SM. Short-term effect of simulated salt marsh restoration by sand-amendment on sediment bacterial communities. PLoS One 2019;14:e0215767.

106. Zamkovaya T, Foster JS, de Crécy-Lagard V, Conesa A. A network approach to elucidate and prioritize microbial dark matter in microbial communities. ISME J. 2020151 2020;15:228-44.

107. Klatt CG, Wood JM, Rusch DB, Bateson MM, Hamamura N, Heidelberg JF, et al. Community ecology of hot spring cyanobacterial mats: predominant populations and their functional potential. ISME J. 201158 2011;5:1262-78.

108. Bairey E, Kelsic ED, Kishony R. High-order species interactions shape ecosystem diversity. Nat. Commun. 2016 71 2016;7:1-7.

994

109. Chiang YS, Chen YW, Chuang WC, Wu Cl, Wu C Te. Triadic balance in the brain: Seeking 
brain evidence for Heider's structural balance theory. Soc. Networks 2020;63:80-90.

110. Zhang Q, Acuña JJ, Inostroza NG, Duran P, Mora ML, Sadowsky MJ, et al. Niche Differentiation in the Composition, Predicted Function, and Co-occurrence Networks in Bacterial Communities Associated With Antarctic Vascular Plants. Front. Microbiol. 2020;0:1036.

111. Venturelli OS, Carr A V, Fisher G, Hsu RH, Lau R, Bowen BP, et al. Deciphering microbial interactions in synthetic human gut microbiome communities. Mol. Syst. Biol. 2018;14:e8157.

112. Jiao J-Y, Liu L, Hua Z-S, Fang B-Z, Zhou E-M, Salam N, et al. Microbial dark matter coming to light: challenges and opportunities. Natl. Sci. Rev. 2021;8:2021.

113. Bolhuis H, Cretoiu MS, Stal LJ. Molecular ecology of microbial mats. FEMS Microbiol. Ecol. 2014;90:335-50.

114. Tropini C, Moss EL, Merrill BD, Ng KM, Higginbottom SK, Casavant EP, et al. Transient Osmotic Perturbation Causes Long-Term Alteration to the Gut Microbiota. Cell 2018;173:1742-1754.e17.

\section{Supplementary Material}

Fig S1. SparCC parameter selection. SparCC parameters were set based on our most complex network: Archean Domes. We ran SparCC varying A. the number of iterations from 10 to $100, \mathbf{B}$. the exclusion number from 10 to 100 and $\mathbf{C}$. the exclusion threshold from 0.1 to 0.9 . We chose the final values based on the stabilization of the number of edges found, such that the final values used for our databases were: 50 iterations, 10 exclusion number and 0.1 for exclusion threshold.

Fig S2. Kombucha topology comparison. A. Distributions obtained from simulated random (red), scale-free (blue) and small-world (green) networks and its comparison to the metrics found in the kombucha network for: degree variance, modularity, average path length, small-world index, and clustering coefficient. B. Degree distribution of the kombucha network. We also show the comparison of the kombucha CCDF with a random network CCDF, a small-world network, and a scale-free network.

Fig S3. Bar plot for community analysis composition (Louvain groups) from the kombucha Network. Community ID is shown in the x-axis.

Fig S4. Bar plot for community analysis composition (HDBSCAN groups) from the kombucha Network. Cluster ID is shown in the x-axis.

Fig S5. Archean Domes topology comparison. A. Distributions obtained from simulated random (red), scale-free (blue) and small-world (green) networks and its comparison to the metrics found in the Archean Domes network for: degree variance, modularity, average path length, small-world index, and clustering coefficient. B. Degree distribution of the Archean Domes network. We also show the comparison of the kombucha CCDF with a random network CCDF, a small-world network, and a scale-free network. 
Fig S6. Bar plot for community analysis composition (Louvain groups) from the Archean Domes Network. Community ID is shown in the x-axis. Unassigned bacteria and not annotated sequences are grouped in the NA category.

Fig S7. Bar plot for community analysis composition (HDBSCAN groups) from the Archean Domes Network. Cluster ID is shown in the x-axis. Unassigned bacteria and not annotated sequences are grouped in the NA category.

Table S1. Network metrics for three canonical topologies. Large scale metrics of three simulated networks with a random, scale-free and small world topology.

Table S2. Main metrics and network properties of the kombucha co-occurrence network.

Table S3. Centrality measures from potential key players in kombucha network. Degree, closeness, betweenness, and PageRank centrality was calculated for the top 5 ASV respectively. If a given ASV was not among the top 5 in a centrality measure, the value is reported as NA.

Table S4. Community analysis (Louvain groups) metrics on kombucha network. For each community, total nodes, diameter, clustering coefficient, and average shortest path were calculated. Clusters are ordered by increasing density.

Table S5. Community analysis (HDBSCAN groups) metrics on kombucha network. For each community, total nodes, diameter, clustering coefficient, and average shortest path were calculated. Clusters are ordered by increasing density.

Table S6. Network robustness analysis for the kombucha network. Random, by groups (Genus), and by degree centrality percolation simulations were performed on the Louvain groups. For the percolation by groups, only Komagataeibacter and is shown.

Table S7. Main metrics and network properties of the Archean Domes co-occurrence network.

Table S8. Centrality measures from potential key players in Archean Domes network. Degree, closeness, betweenness, and PageRank centrality was calculated for the top 10 ASV respectively. If a given ASV was not among the top 10 in a centrality measure, the value is reported as NA.

Table S9. Community analysis (Louvain groups) metrics on Archean Domes network. For each community, total edges, total nodes, average degree, clustering coefficient, and density were calculated. Clusters are ordered by increasing density.

Table S10. Community analysis (HDBSCAN groups) metrics on Archean Domes network. For each community, total edges, total nodes, average degree, clustering coefficient, and density were calculated. Clusters are ordered by increasing density. 
bioRxiv preprint doi: https://doi org/10.1101/2021.11.11.468289; this version posted November 11, 2021. The copyright holder for this preprint (which was not certified by peer review) is the author/funder, who has granted bioRxiv a license to display the preprint in perpetuity. It is made available under aCC-BY 4.0 International license.

1085 Table S11. Network robustness analysis from the Archean Domes network. Random, by 1086 groups (Phylum), and by degree centrality percolation simulations were performed on the Louvain groups. For the percolation by groups, only Cyanobacteria percolation is shown. 


\begin{tabular}{|c|c|c|c|}
\hline & RANDOM & SCALE-FREE & SMALL-WORLD \\
\hline Graph & $\sum_{0}$ & $\int_{0}^{0}=0$ & $\int_{0}^{\infty}$ \\
\hline $\begin{array}{l}\text { Degree } \\
\text { distribution }\end{array}$ & Poisson distribution & Power-law distribution & Power-law distribution \\
\hline $\begin{array}{l}\text { Mini } \\
\text { description }\end{array}$ & $\begin{array}{l}\text { Nodes are randomly } \\
\text { connected with } \\
\text { probability } p \text {, thus most } \\
\text { nodes have equal number } \\
\text { of degrees. }\end{array}$ & $\begin{array}{l}\text { A small number of nodes } \\
\text { has a high degree (i.e. are } \\
\text { connected with many } \\
\text { nodes), whereas the } \\
\text { other nodes have low } \\
\text { degree. }\end{array}$ & $\begin{array}{l}\text { There is a short } \\
\text { average path between } \\
\text { most nodes, similar to } \\
\text { a random, but they } \\
\text { display higher } \\
\text { clustering coefficients. }\end{array}$ \\
\hline
\end{tabular}




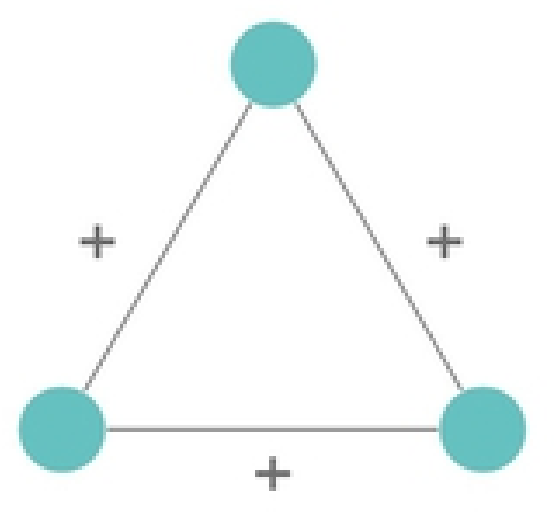

BALANCED TRIANGLES

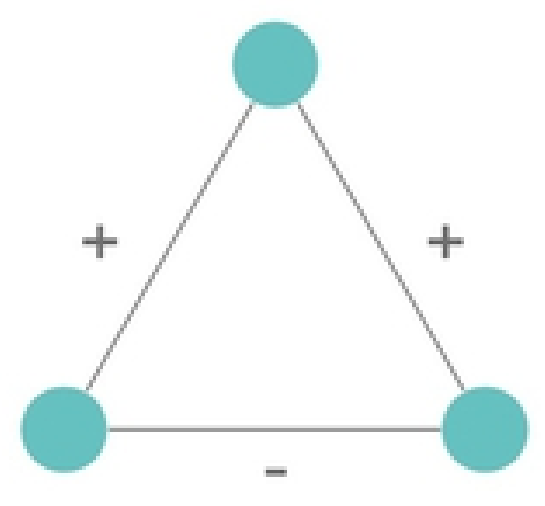

INBALANCED TRIANGLES

Figure 3 

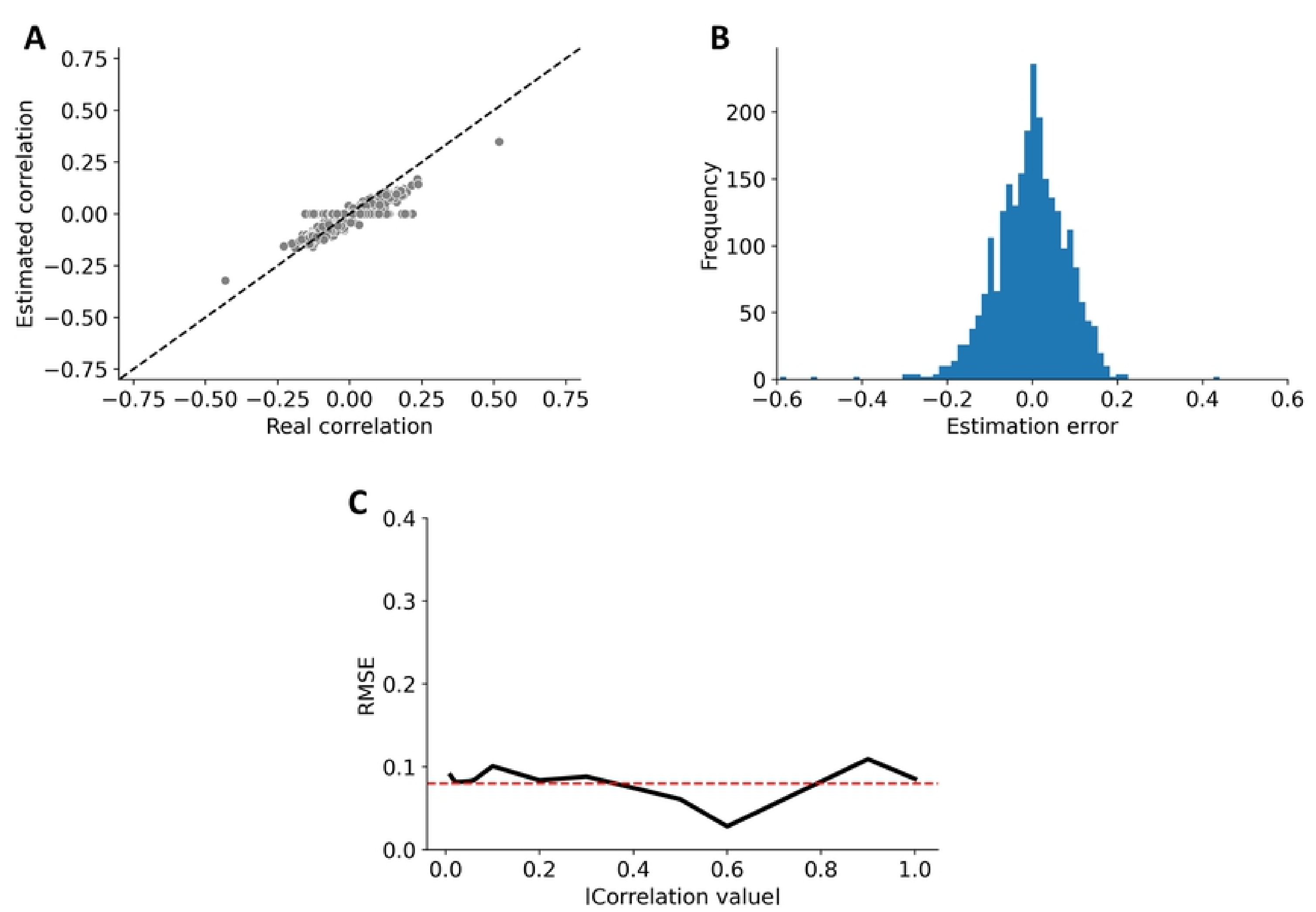

Figure 4 
A

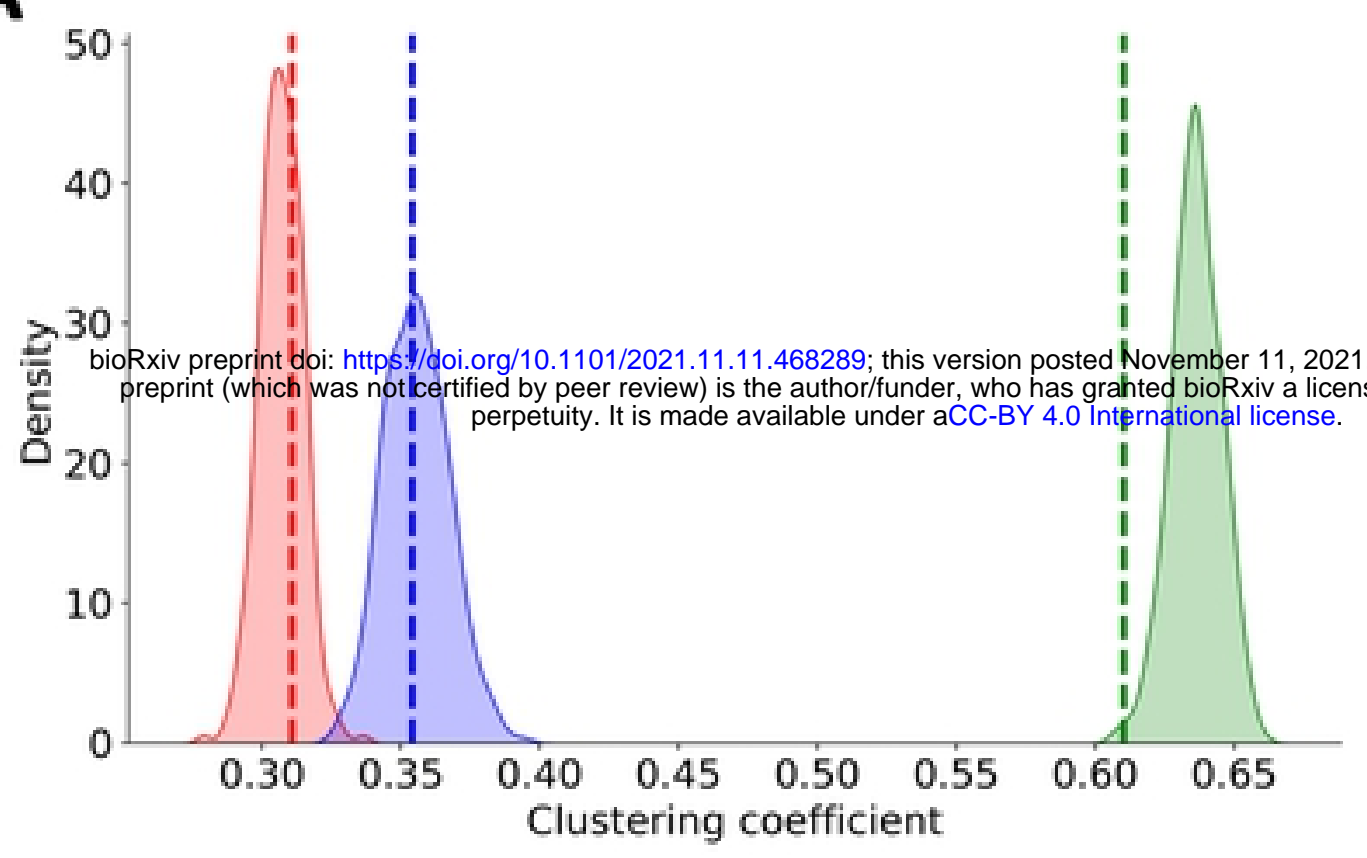

C

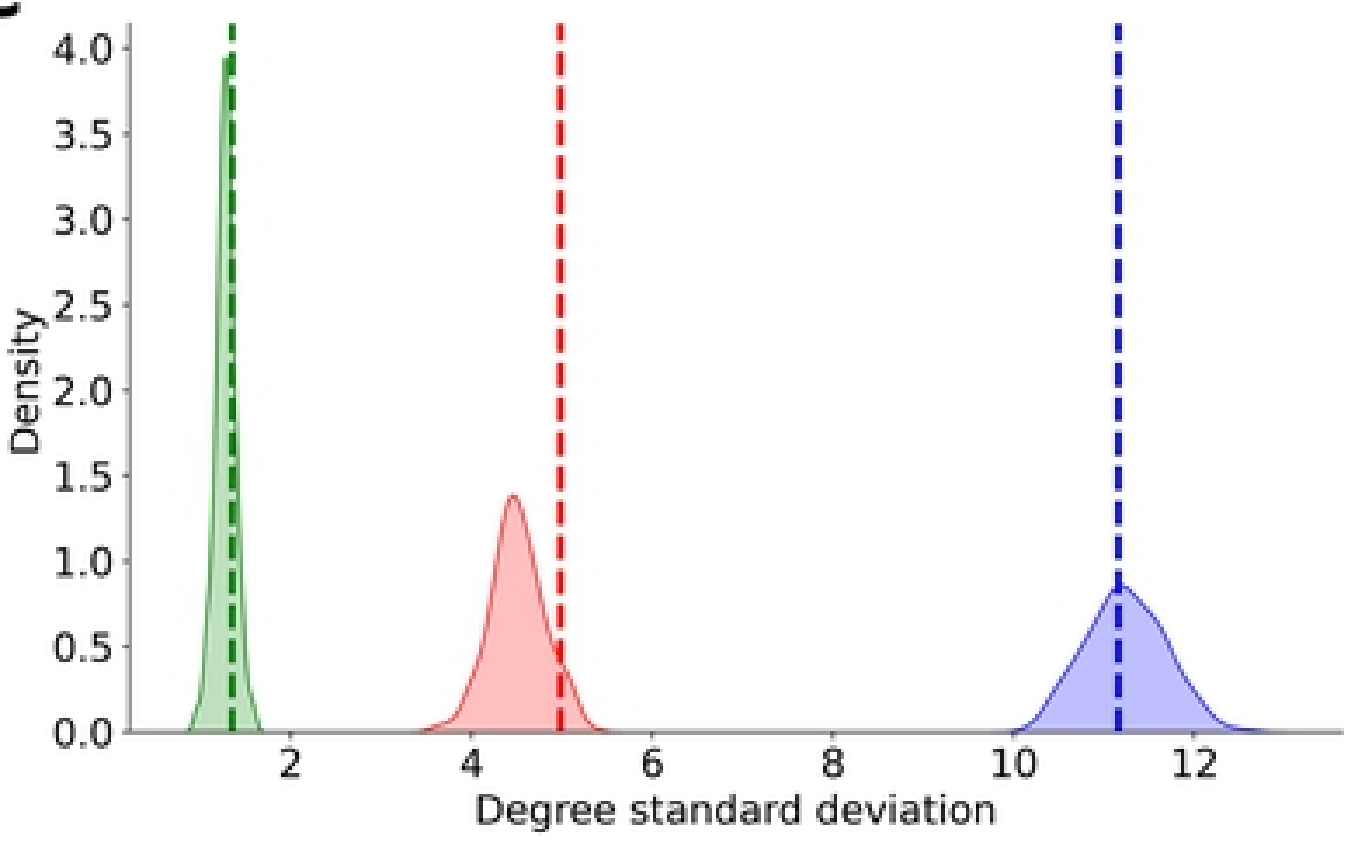

E

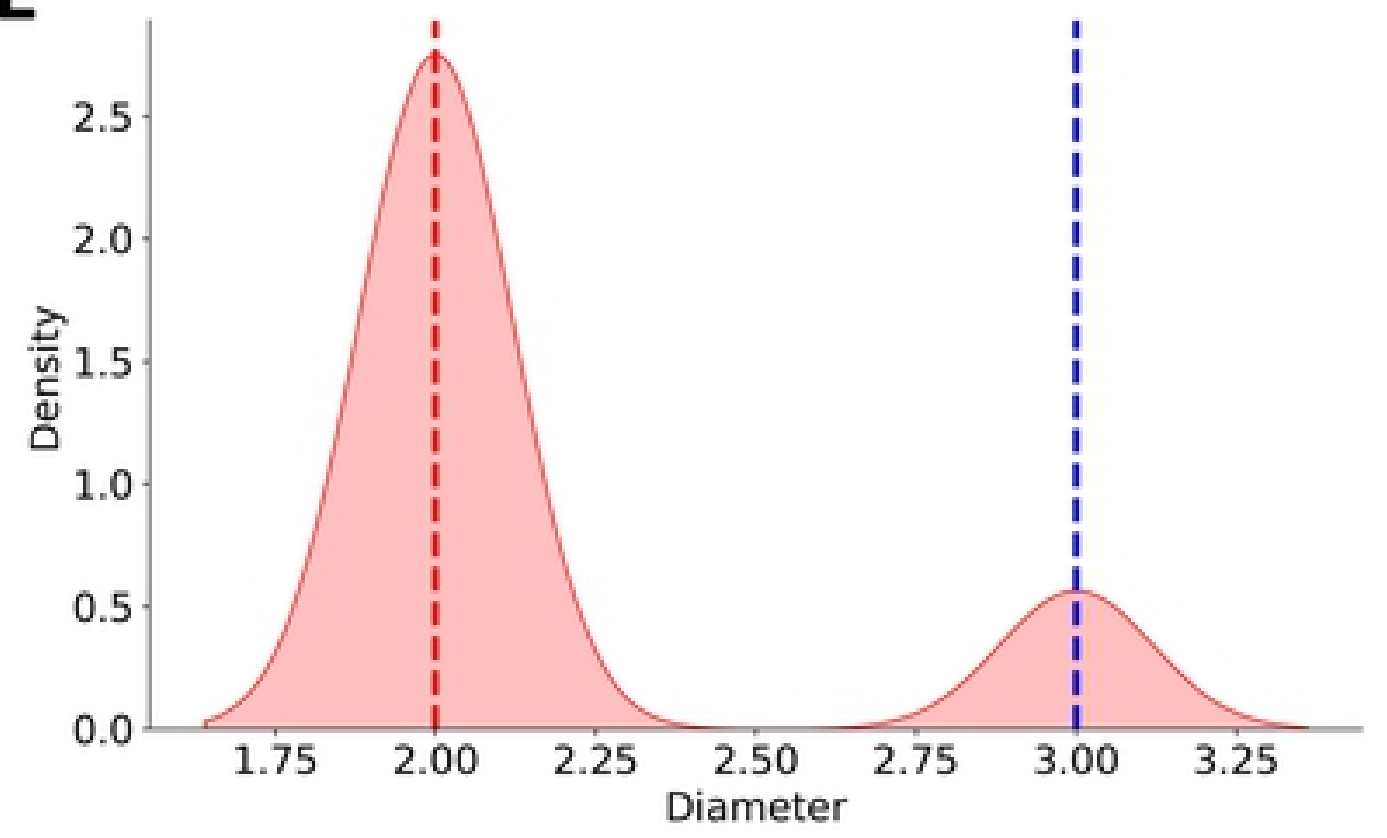

B

fight holder for thi ay the preprint in

○ 100

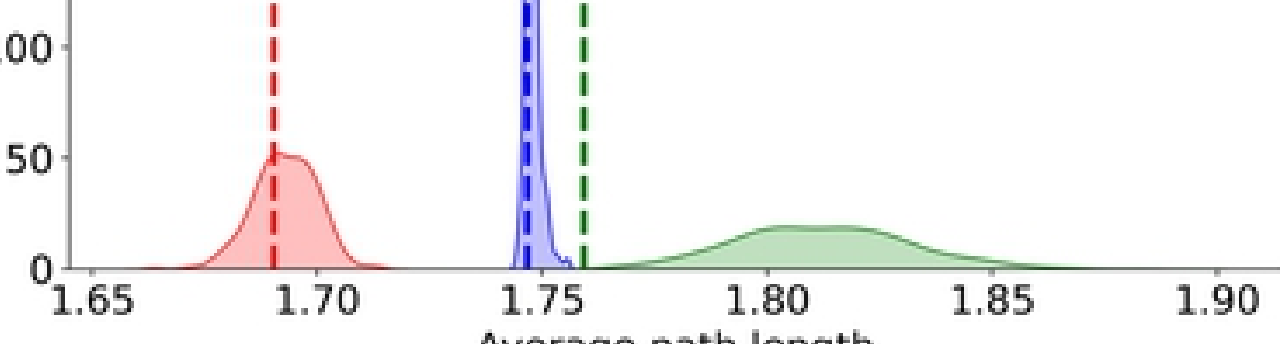

D

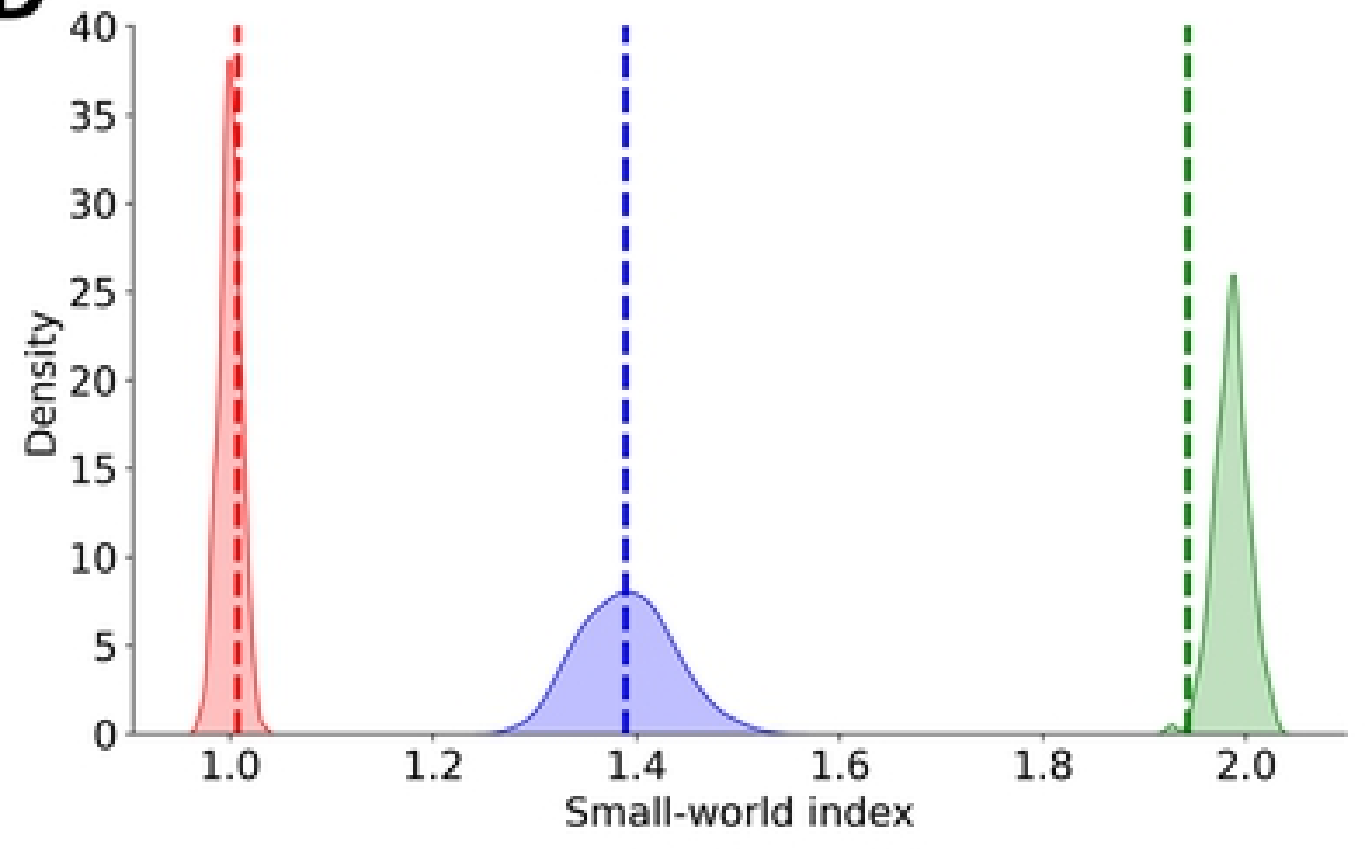

F

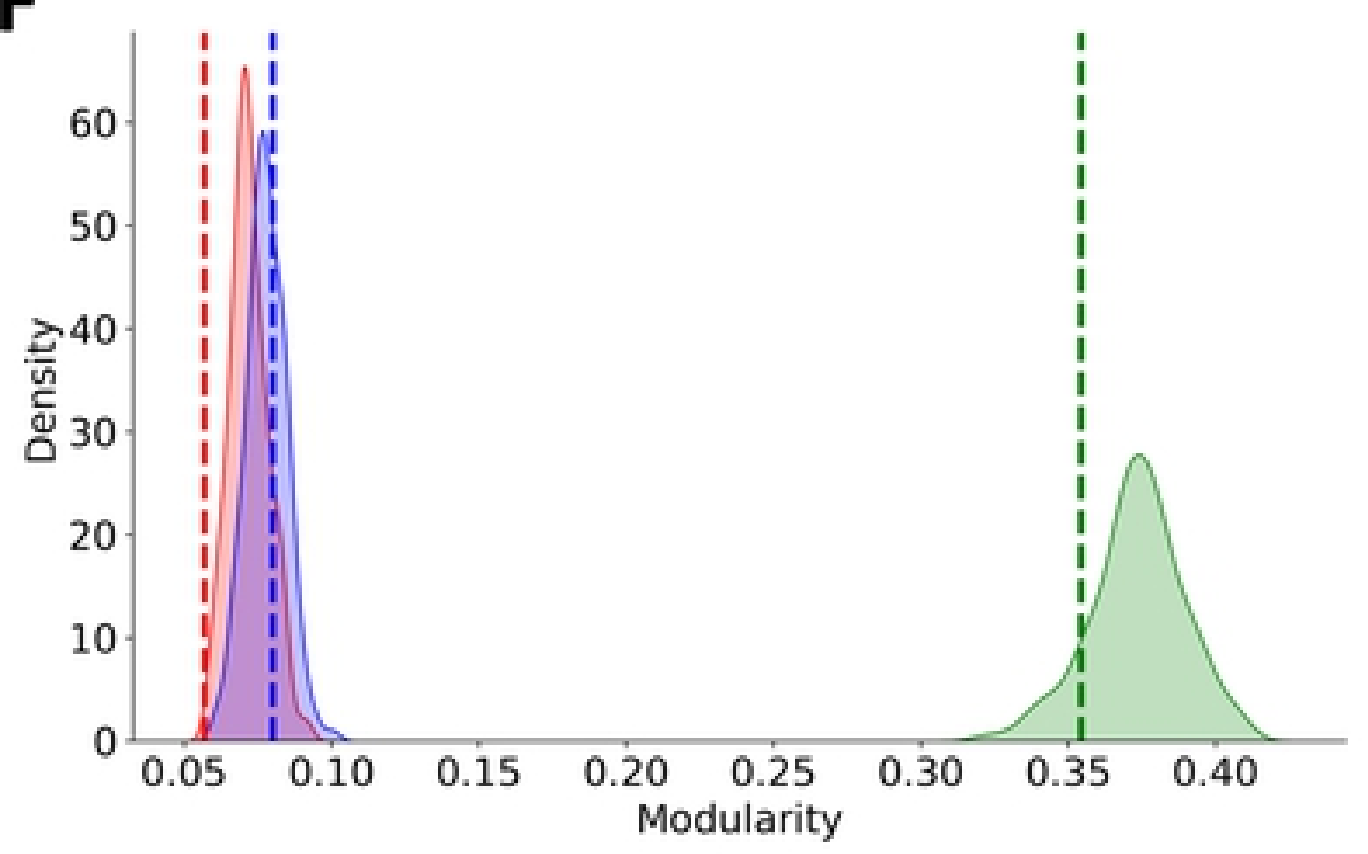

Figure 5 


\section{A. Random}
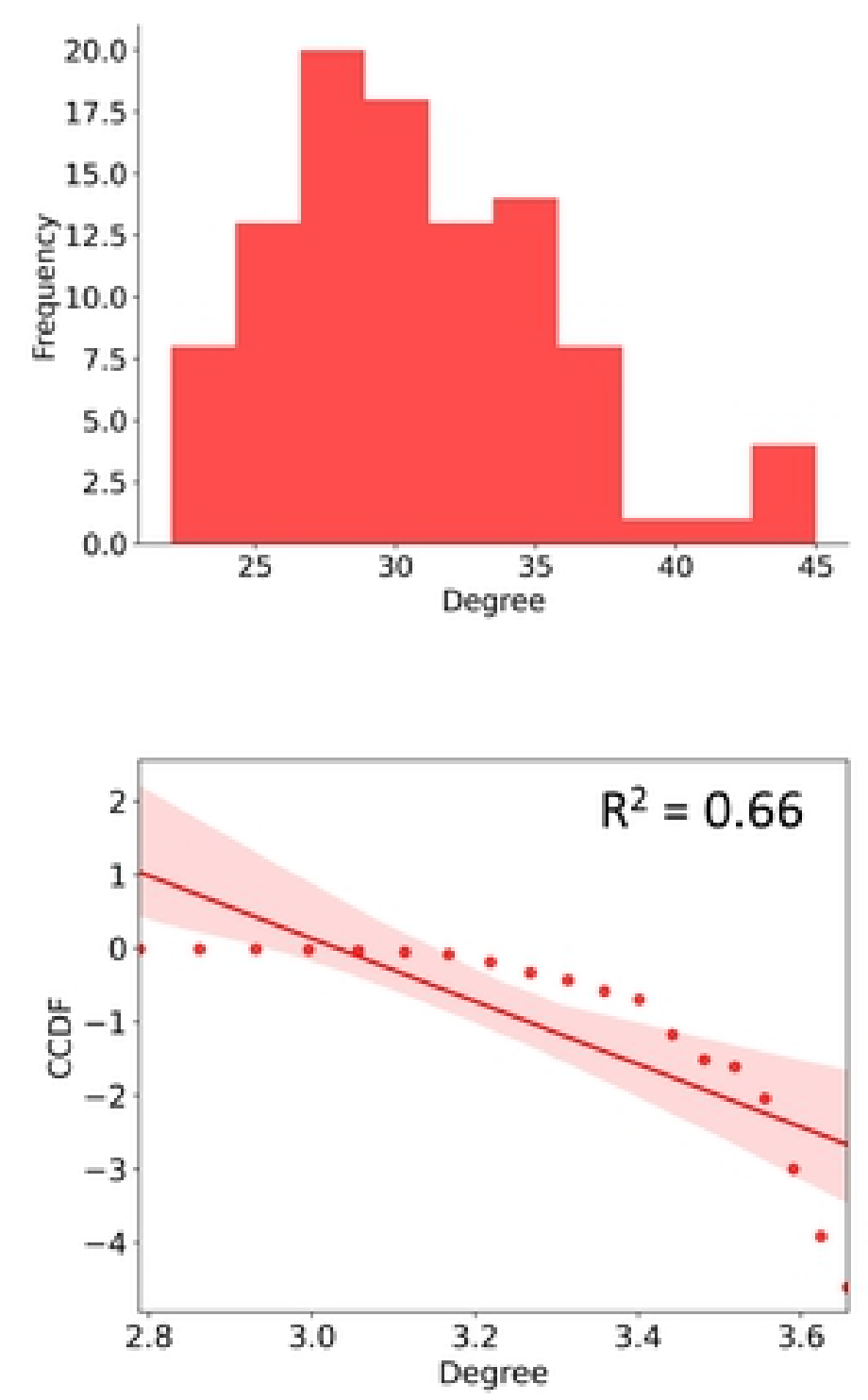

B. Scale-free
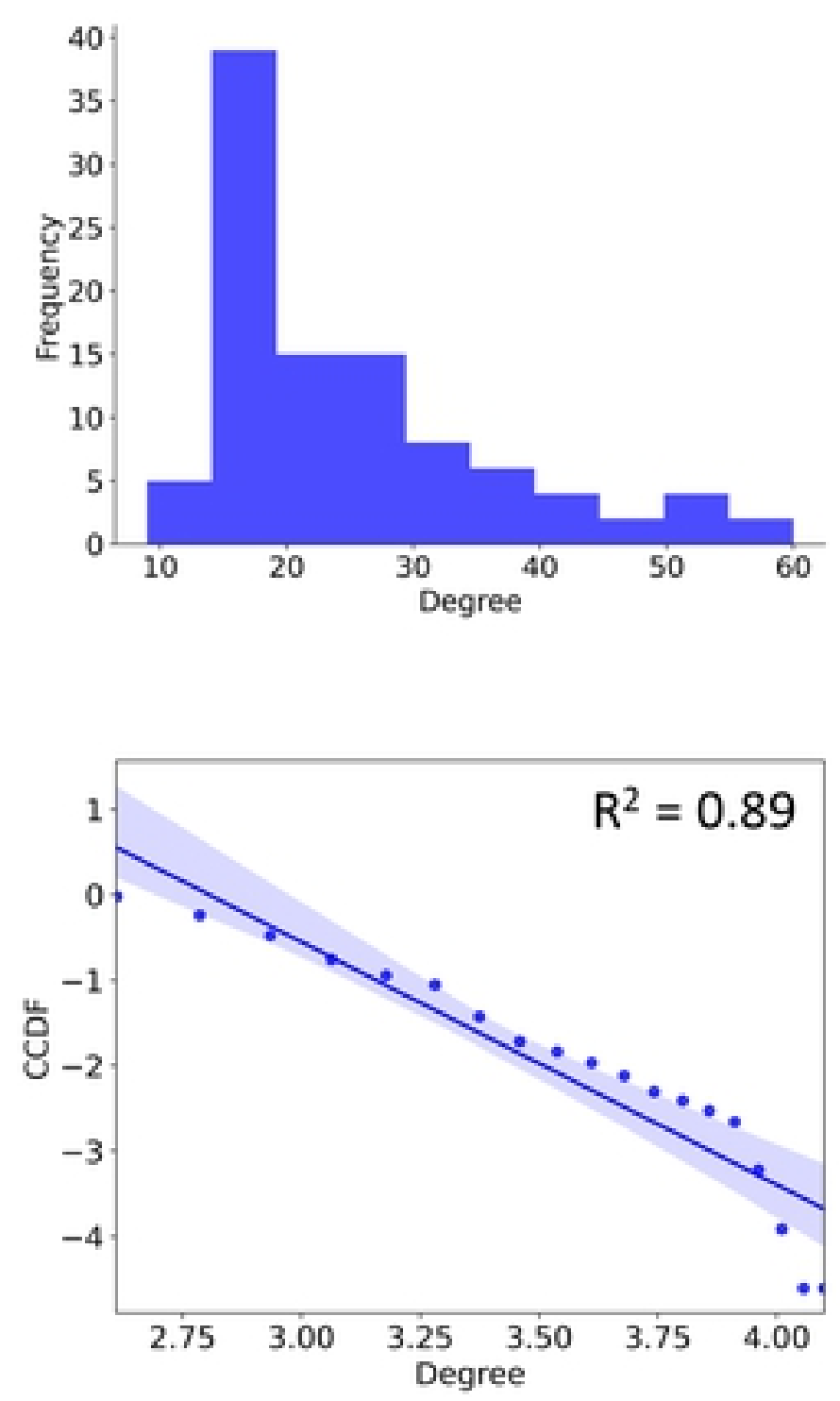

C. Small-world
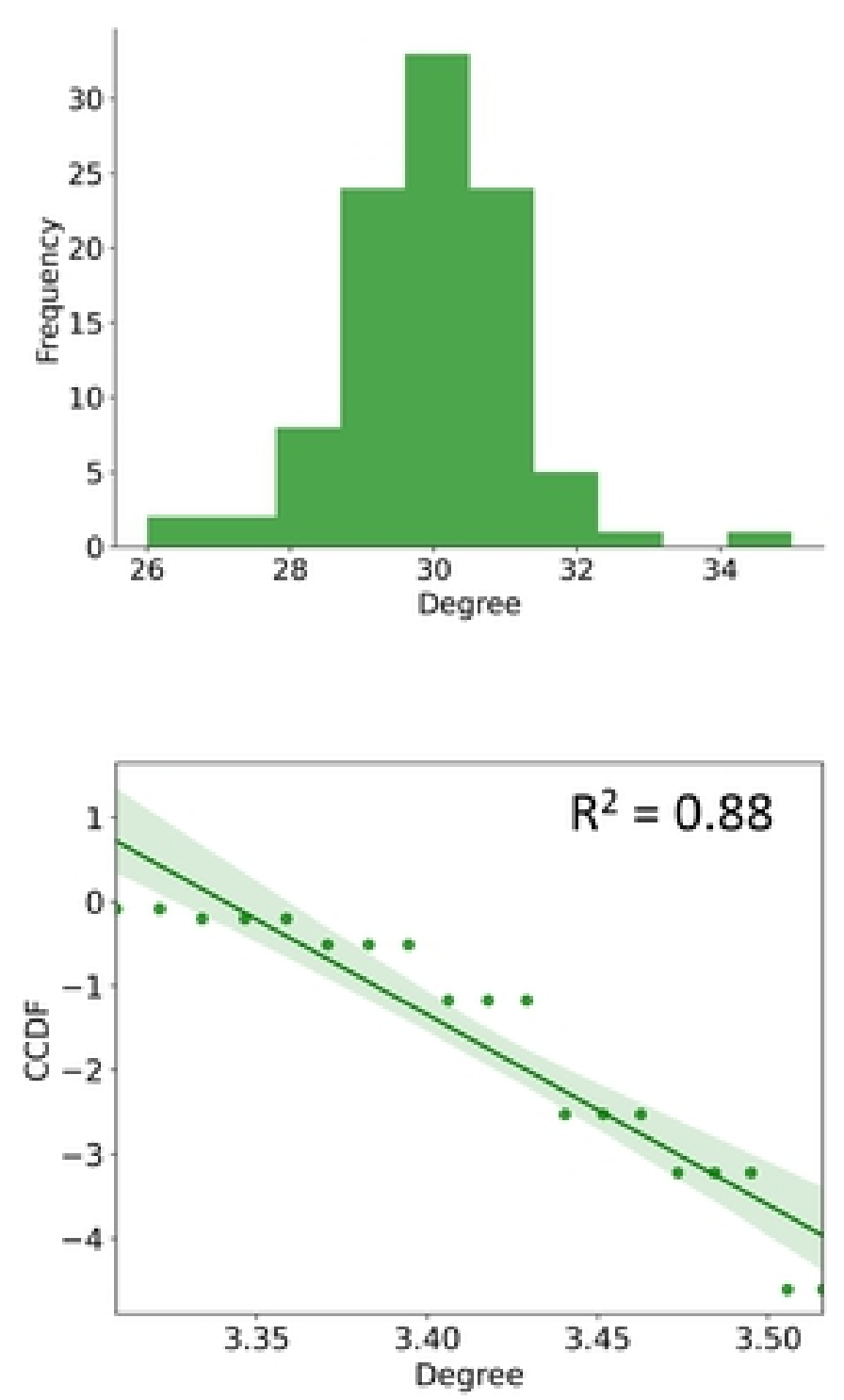

Figure 6 
A

$$
\text { Network - Community }
$$

B Network - HDBSCAN

bioRxiv reprint doit: https://doi.org/10.1101/2021.11.11.468289; this version posted November 11, 2021. The copyright holder for this
reprint (which was not certified by peer review) is the author/funder, who has granted bioRxiv a license to display the preprint in
perpetuity. It is made available under aCC-BY 4.0 International license.

○。

8

$\because$
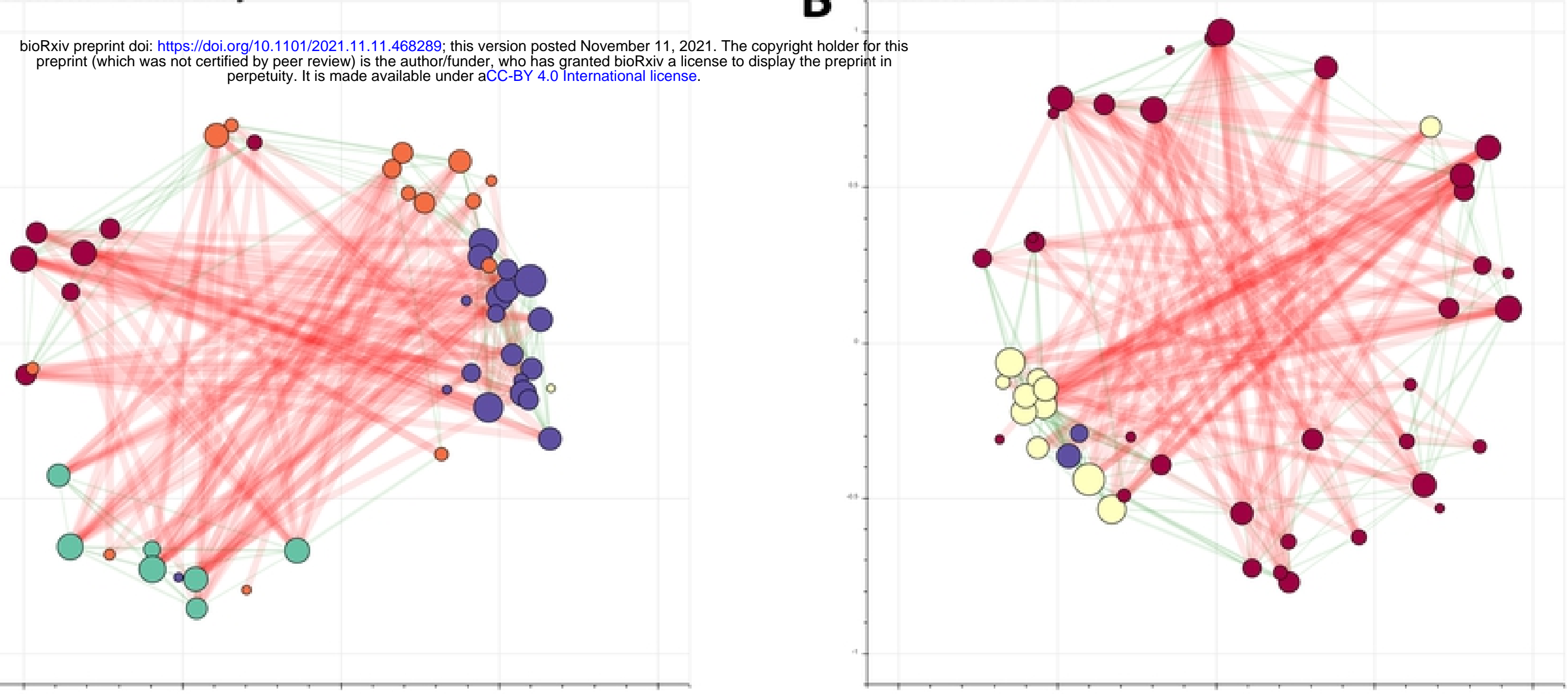

C

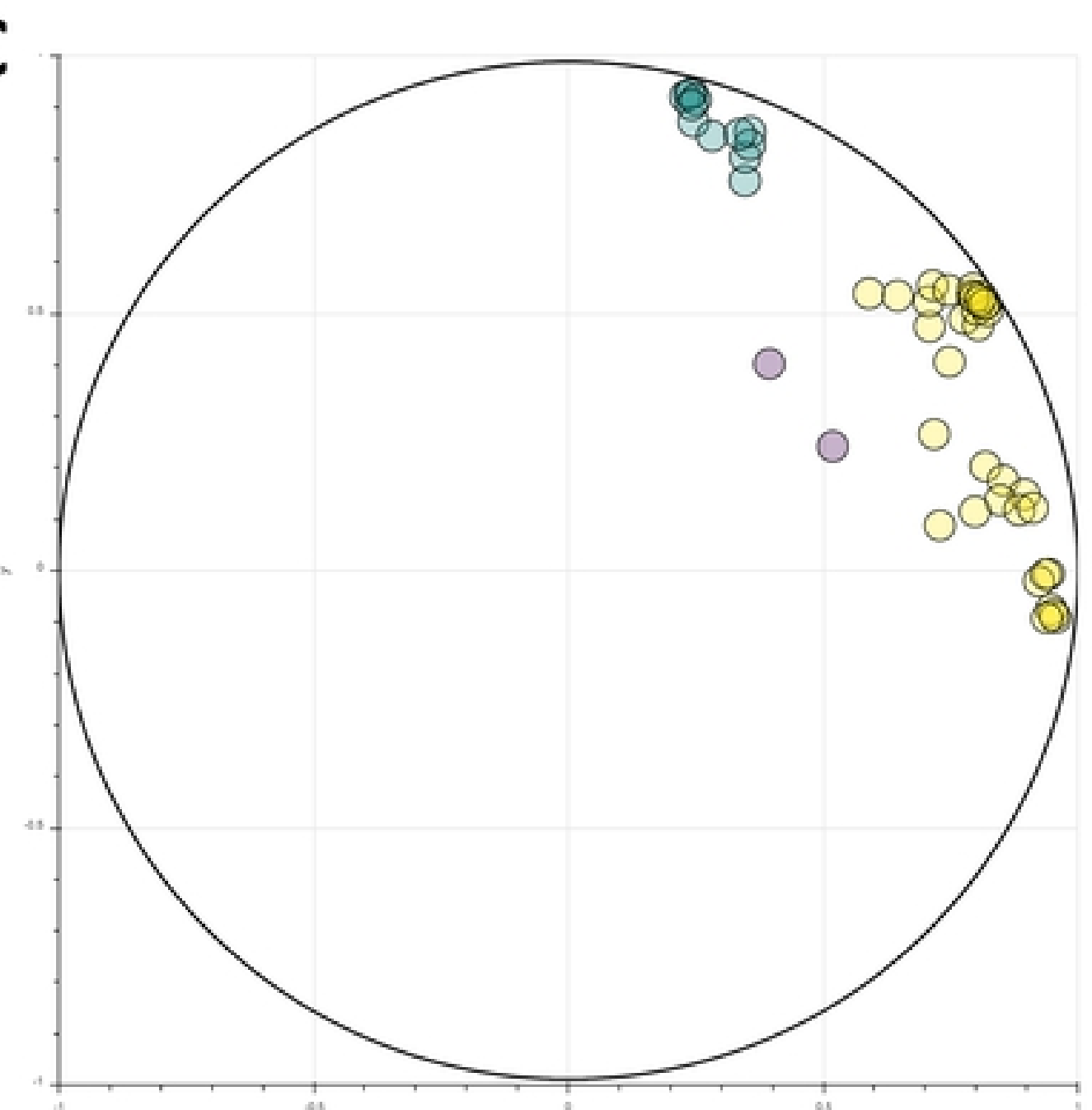

Figure 7 
A Network-Community

B Network-HDBSCAN

bioRxiv preprint doi: https://doi.org/10.1101/2021.11.11.468289; this version posted November 11, 2021. The copyright holder for this

preprint (which was not certified by peer review) is the authorffunder, who has granted bioRxiv a license to display the preprint in
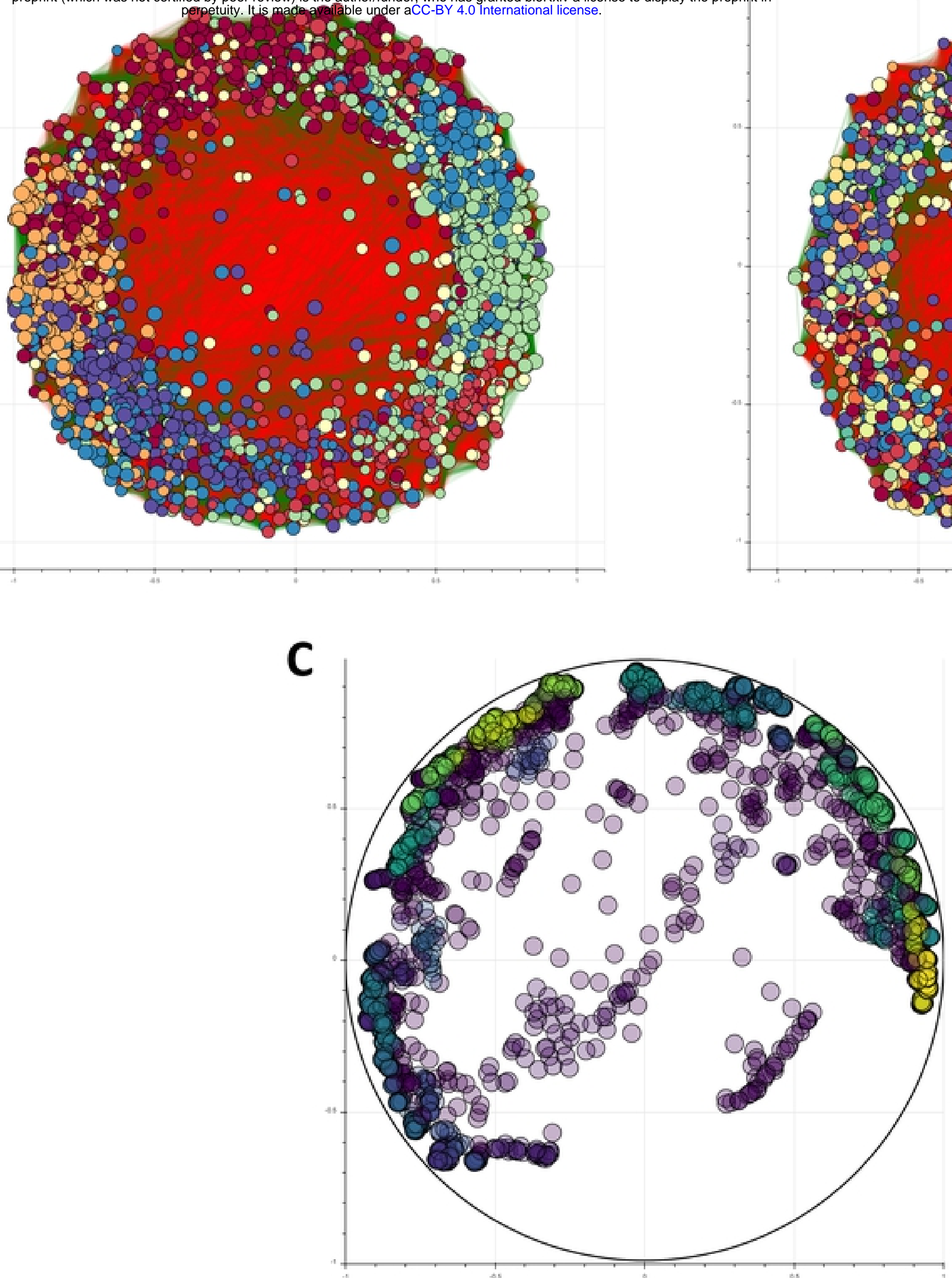

Figure 8 